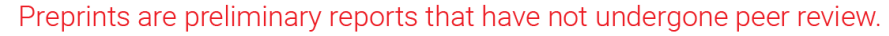 They should not be considered conclusive, used to inform clinical practice, or referenced by the media as validated information. \\ Inflammatory Metabolic Profile of South African Patients with Prostate Cancer
}

\section{Stefano Cacciatore}

International Centre for Genetic Engineering and Biotechnology

\section{Martha Wium}

International Centre for Genetic Engineering and Biotechnology

\section{Cristina Licari}

University of Florence: Universita degli Studi di Firenze

\section{Aderonke Ajayi-Smith}

International Centre for Genetic Engineering and Biotechnology

\section{Lorenzo Masieri}

University of Florence: Universita degli Studi di Firenze

\section{Chanelle Anderson}

International Centre for Genetic Engineering and Biotechnology

\section{Azola Samkele Salukazana}

University of Florence: Universita degli Studi di Firenze

\section{Lisa Kaestner}

University of Cape Town

\section{Marco Carini}

University of Florence: Universita degli Studi di Firenze

\section{Giuseppina M Carbone}

Università della Svizzera Italiana: Universita della Svizzera Italiana

\section{Carlo V. Catapano}

Università della Svizzera Italiana: Universita della Svizzera Italiana

\section{Massimo Loda}

Cornell University

\section{Towia A. Libermann}

Beth Israel Deaconess Medical Center

Luiz Fernando Zerbini (D luiz.zerbini@icgeb.org)

International Centre for Genetic Engineering and Biotechnology https://orcid.org/0000-0003-07369508

\section{Research}


Keywords: Metabolomics, NMR spectroscopy, GlycA, GlycB, histidine, Prostate cancer, Africa

Posted Date: December 8th, 2020

DOI: https://doi.org/10.21203/rs.3.rs-120163/v1

License: (c) (1) This work is licensed under a Creative Commons Attribution 4.0 International License. Read Full License

Version of Record: A version of this preprint was published at Cancer \& Metabolism on August 3rd, 2021. See the published version at https://doi.org/10.1186/s40170-021-00265-6. 
2 Stefano Cacciatore ${ }^{1,2, \dagger}$, Martha Wium ${ }^{1, \dagger}$, Cristina Licari ${ }^{3, \dagger}$, Aderonke Ajayi-Smith ${ }^{1}$, Lorenzo Masieri ${ }^{4,5}$, 3 Chanelle Anderson ${ }^{1}$, Azola Samkele Salukazana ${ }^{6}$, Lisa Kaestner ${ }^{6}$, Marco Carini ${ }^{4}$, Giuseppina M. Carbone $^{7}$, Carlo V. Catapano ${ }^{7,8,9}$, Massimo Loda ${ }^{10,11}$, Towia A. Libermann ${ }^{12}$, Luiz F. Zerbini ${ }^{1 *}$

$6{ }^{1}$ Cancer Genomics Group, International Centre for Genetic Engineering and Biotechnology, Cape Town, 7 South Africa.

$8 \quad 2$ Institute for Reproductive and Developmental Biology, Imperial College, London, United Kingdom.

$9{ }^{3}$ Magnetic Resonance Center (CERM), University of Florence, Sesto Fiorentino, Italy.

$10{ }^{4}$ Department of Urology, Clinica Urologica I, Azienda Ospedaliera Careggi, University of Florence, 11 Florence, Italy.

$12{ }^{5}$ Pediatric Urology Unit, Meyer Children Hospital, University of Florence, Florence, Italy.

$13{ }^{6}$ Division of Urology, University of Cape Town, Groote Schuur Hospital, Cape Town, South Africa.

$14{ }^{7}$ Institute of Oncology Research (IOR), Università della Svizzera italiana, Bellinzona, Switzerland.

$15{ }^{8}$ Swiss Institute of Bioinformatics (SIB), Lausanne, Switzerland.

169 Department of Oncology, Faculty of Biology and Medicine, University of Lausanne, Lausanne, 17 Switzerland.

$18{ }^{10}$ Department of Oncologic Pathology, Dana-Farber Cancer Institute, Boston, Massachusetts.

$19{ }^{11}$ Department of Pathology and Laboratory Medicine, Weill Cornell Medicine, New York, New York.

2012 BIDMC Genomics, Proteomics, Bioinformatics and Systems Biology Center, Beth Israel Deaconess 21 Medical Center and Harvard Medical School, Boston, Massachusetts.

$22 \uparrow$ Contributed equally to this work.

$23 *$ Corresponding author 


\section{Abstract}

25 Background: Men with African ancestry are more likely to develop aggressive prostate cancer (PCa) and to die from this disease. A distinct genomic landscape associated with specific ethnic groups may lead to different metabolic adaptations and inflammatory responses that permit tumor cells to proliferate and to grow. We hypothesize that a higher risk of lethal PCa in men with African ancestry may be associated with high level of systemic inflammation.

Methods: In this study, plasma samples were profiled from a cohort of 41 South African men with PCa using Nuclear Magnetic Resonance (NMR) spectroscopy. A total of 41 features, including metabolites, lipid classes, total protein, and the inflammatory NMR markers, GlycA and GlycB were quantified from each NMR spectrum. The Bruker's B.I.-LISA protocols were used to characterize 114 parameters related to the lipoproteins, such as HDL, LDL and VLDL, and their relative subclasses. The unsupervised KODAMA method was used to stratify the patients of our cohort based on their metabolic profile.

Results: We found that the plasma of patients with very high risk, aggressive $\mathrm{PCa}$ and high level of Creactive protein have a peculiar metabolic phenotype (metabotype) characterized by extremely high levels of GlycA and GlycB. The inflammatory processes linked to the higher level of GlycA and GlycB are 39 characterized by a deep change of the plasma metabolome that leads the stratification of patients with PCa.

40 We also identified a not previously relationship between high values of very-high density lipoprotein and 41 low level of GlycB in a different metabotype of patients characterized by lower risk PCa.

42 Conclusions: Systemic inflammation plays a role in the metabolic profile of cancer. This study advances 43 our understanding of the relationship between metabolome and systemic inflammation in the context of 44 PCa and opens the door to a totally innovative approach for biomarker discovery and to the development 45 of new therapies aiming to reduce the systemic inflammation in these patients.

46 Keywords: Metabolomics, NMR spectroscopy, GlycA, GlycB, histidine, Prostate cancer, Africa 


\section{Abbreviations:}

49 AA, African American; ADT, androgen-deprivation therapy; APP, acute-phase protein; BO, bilateral 50 orchidectomy; CRP, C-reactive protein; CRPC, castration-resistant prostate cancer; CSPC, castration51 sensitive prostate cancer EA, European American; FDR, false discovery rate; HDL, high-density 52 lipoprotein; IDL, intermediate-density lipoprotein; IL, interleukin; IFN, type I interferon; LDL, low-density 53 lipoprotein; metabotype, metabolic phenotype; mCRPC, metastatic castration-resistant prostate cancer; 54 NCCN, National Comprehensive Cancer Network; NMR, nuclear magnetic resonance; NOESY, Nuclear 55 Overhauser Effect SpectroscopY; PCa, prostate cancer; PLS, partial least-squares; PSA, prostate-specific 56 antigen; Q2, predictive ability parameter; R2, fit parameter; rho, correlation coefficient VLDL, very-low57 density lipoprotein. 
59 Prostate cancer (PCa) is the second most frequent cancer diagnosis made in men and the fifth leading cause

60 of death worldwide [1]. Advancing age, family history of PCa and African ancestry are among established 61 risk factors [2]. PCa in men of African descent tends to have more aggressive phenotypes compared with 62 other ethnicities [3]. Studies investigating the possible role of genetic susceptibility in affecting PCa 63 disparities are often limited to the African American (AA) population. Comparing to European American 64 (EA) men, AA men have two-thirds higher incidence and two-fold greater risk of dying of $\mathrm{PCa}[3]$. The 65 hypothesis that ethnic disparity may be related to biological differences in PCa phenotype is supported by 66 recent studies conducted in Southern Africa. Men from Southern Bantu populations have a 2.1-fold and 67 4.9-fold greater risk than AAs for presenting at diagnosis a PCa with Gleason score $\geq 8$ and prostate-specific 68 antigen (PSA) $\geq 20 \mathrm{ng} / \mathrm{mL}$ [4]. Recently, a pilot Whole-Genome Sequencing study conducted on six 69 African prostate tumors indicated a doubling of the mutational burden in African men compared to men of 70 European ancestry [5]. Disparities in PCa risk and aggressiveness across different ethnicities are poorly 71 understood and likely influenced by genetic factors as well as difficulties to access medical resources in 72 Africa [6].

73 The South African population is a unique blend of African and non-African ancestry [7]. Its ethnic diversity 74 represents both a challenge and an opportunity for biomedical research [8]. Currently, the major 75 ethnolinguistic groups in South Africa are Black southeastern Bantu-speakers, an admixed population 76 referred to as Coloured [9], Whites of European origin (8.9\%), and an Indian population originating from 77 the Indian sub-continent $(2.5 \%)[7]$.

78 Lately, more effort has been put on exploring the genetic factors contributing to PCa in men of African 79 ancestry [10-13]. Several genes have been identified as differentially methylated or expressed in PCa 80 between men of European or African ancestry [14]. The number of studies linking the association of one 81 or multiple single nucleotide polymorphisms in inflammation-related pathways to PCa risk has greatly 82 increased $[15,16]$. Since inflammation may contribute to PCa development and progression to advanced 
83 metastatic disease [17], polymorphisms in immune-related genes could at least partially explain the 84 different incidence and mortality of PCa in African men [18]. AA men, compared to EA men, show an 85 increased incidence of inflammation in biopsy specimens [19] and increased expression of immune-related 86 genes in tumor tissues [20]. A distinct genomic landscape of PCa and immune-related genes associated 87 with specific ethnic groups may lead to different metabolic adaptations that permit tumor cells to proliferate. 88 While metabolic reprogramming is known to play a significant role in both PCa initiation and progression, 89 inflammation seems to be ignored in most biomarker studies [21], including metabolomic studies.

90 Chronic inflammation has a strong impact on the human metabolome [21]. Metabolic analysis may 91 illuminate systemic metabolic consequences of inflammation and provide novel targets for intervention. 92 Nuclear magnetic resonance (NMR) spectroscopy is a particularly powerful technique when applied to the 93 high-throughput analysis of biofluids such as blood [22] which can be collected with minimal impact on 94 the participant. NMR-based metabolomics is a straightforward and useful method for the qualitative and 95 quantitative analysis of a wide range of components in blood samples, including low-molecular-weight 96 metabolites and lipoproteins (different for size and composition) [23]. Moreover, NMR spectroscopy 97 allows the detection in plasma of signals arising from the glycosylation of circulating acute-phase proteins 98 (APPs), such as fibrinogen, $\alpha 1$-antichymotrypsin, haptoglobin-1, $\alpha 1$-antitrypsin, complement C3 and $\alpha 1$ 99 acid glycoprotein [24].

100 The carbohydrate portions of glycoproteins containing $\mathrm{N}$-acetylglucosamine and $\mathrm{N}$-acetylgalactosamine 101 (hereinafter referred to as GlycA) and N-acetylneuraminic acid (a.k.a., sialic acid; hereinafter referred to as 102 GlycB) moieties are visible as two distinct NMR signals. GlycA and GlycB levels have been associated 103 with common markers of inflammation such as C-reactive protein (CRP), fibrinogen, interleukin (IL)-6, 104 tumor necrosis factor-alpha, lipoprotein-associated phospholipase $\mathrm{A}_{2}$ and serum amyloid A [25-28]. Similar 105 to CRP, GlycA is a marker of chronic inflammation [27]; despite the similarity, GlycA and CRP likely 106 capture different aspects of the inflammatory response [29]. CRP is an "early" APP and the proteins that 107 contribute the most to the GlycA and GlycB signal rise later in the acute phase response [30]. In response 
108 to acute and chronic inflammatory stimuli both the concentrations of APPs and their glycan structures are 109 modified [30].

110 This study, to our knowledge, is the first to metabolic profile PCa in men from Africa. Here, we used NMR 111 spectroscopy to quantify a panel of 41 signals, including metabolites, lipid groups, proteins, and the

112 inflammatory markers GlycA and GlycB. Moreover, we used an advanced lipoprotein test based on NMR 113 spectroscopy to characterize the lipoprotein subclasses in each sample. For the first time, we reported the 114 relationship between these inflammatory biomarkers (i.e., GlycA and GlycB) and the most aggressive PCa 115 cases. We provide a clear snapshot of the metabolic alterations during the inflammatory process in PCa 116 paving the way to a better understanding of the metabolic changes occurring in PCa.

\section{Methods}

118 Patients

119 Participants were recruited from the Urological clinics of Groote Schuur, Eerste Rivier and New Somerset 120 Hospitals in Cape Town, South Africa. Patients scheduled to undergo transurethral resection of the prostate 121 or prostatectomy were enrolled. The diagnosis of PCa was confirmed by histopathologic examinations. The 122 protocol (*HREC454/2012*) was approved by the Human Research Ethics Committee of the Faculty of 123 Health Science, University of Cape Town, South Africa. Written consent was obtained from all the 124 participants before $5 \mathrm{~mL}$ of blood was collected in Vacuette ${ }^{\circledR}$ EDTA tube by medical staff. Blood plasma 125 was separated by centrifugation $\left(1000 \mathrm{~g}\right.$ for $10 \mathrm{~min}$ at $\left.4{ }^{\circ} \mathrm{C}\right)$ and stored at $-80^{\circ} \mathrm{C}$.

\section{$126 \quad$ NMR sample preparation and analysis}

127 Plasma samples were thawed at room temperature. An aliquot of $350 \mu \mathrm{L}$ of a phosphate sodium buffer $128\left(70 \mathrm{mM} \mathrm{Na}_{2} \mathrm{HPO}_{4} ; 20 \%\right.$ (v/v) ${ }^{2} \mathrm{H}_{2} \mathrm{O} ; 6.1 \mathrm{mM} \mathrm{NaN}_{3} ; 4.6 \mathrm{mM}$ sodium 3-trimethylsilyl [2,2,3,3- $\left.{ }^{2} \mathrm{H}_{4}\right]-$ 129 propionate; $\mathrm{pH}$ 7.4) was added to $350 \mu \mathrm{L}$ of each sample. The mixture was homogenized by vortexing for $13030 \mathrm{~s}$, before $600 \mu \mathrm{L}$ of this mixture was transferred into a $5 \mathrm{~mm}$ NMR tube for analysis. 
131 For each plasma sample, one-dimensional ${ }^{1} \mathrm{H}-\mathrm{NMR}$ spectra were acquired on a Bruker $600 \mathrm{MHz}$ 132 spectrometer (Bruker BioSpin) operating at $600.13 \mathrm{MHz}$ proton Larmor frequency and equipped with a $1335 \mathrm{~mm}$ PATXI 1H-13C-15N and 2H-decoupling probe including a $\mathrm{z}$-axis gradient coil, an automatic tuning134 matching and an automatic and refrigerated sample changer (SampleJet). A BTO 2000 thermocouple was 135 used at the level of approximately $0.1 \mathrm{~K}$ on the sample to stabilize the temperature. Before starting 136 measurements, samples were kept inside the NMR probe head for at least 5 minutes to equilibrate 137 temperature at $310 \mathrm{~K}$. The standard Nuclear Overhauser Effect SpectroscopY (NOESY) presat pulse 138 sequence (noesygppr1d.comp; Bruker BioSpin) was used to detect both signals of small metabolites and 139 high molecular weight macromolecules. Parameters of the experiment were: 32 scans, 98304 data points, a 140 spectral width of $18028.846 \mathrm{~Hz}$, an acquisition time of $2.73 \mathrm{~s}$, a relaxation delay of $4 \mathrm{~s}$ and a mixing time 141 of $0.01 \mathrm{~s}$. Transformed spectra were automatically corrected for phase and baseline distortions using 142 Topspin 3.2 (Bruker BioSpin) and then automatically calibrated to the anomeric proton signal of $\alpha$-glucose 143 at $5.24 \mathrm{ppm}$.

\section{Molecular profiling and lipoprotein quantification}

145 Lipoprotein parameters were estimated on NOESY spectra according to Bruker's B.I.-LISA protocols 146 (Bruker IVDr Lipoprotein subclass analysis) [31]. Information related to the main very-low-density 147 lipoprotein (VLDL), low-density lipoprotein (LDL), intermediate-density lipoprotein (IDL), and high148 density lipoprotein (HDL) classes and to their subclasses were extrapolated. In detail, information was 149 extracted of five VLDL subclasses (from VLDL-1 to VLDL-5), six LDL sub-classes (from LDL-1 to LDL150 6), and four HDL-subclasses (HDL-1 to HDL-4) sorted according to increasing density and decreasing size.

151 For each class and subclass, calculated data consist of concentrations of lipids, i.e., cholesterol, free 152 cholesterol, phospholipids, and triglycerides. Instead, concentrations of apolipoproteins Apo-A1 and Apo153 A2 were estimated for HDL class and each relative subclass, while Apo-B concentrations are calculated for 154 VLDL, IDL classes and all LDL subclasses. 
155 Identification of signals was undertaken using the SBASE database in Amix (v3.9.11; Bruker BioSpin,

156 Germany) or available assignments in the literature. The peaks of the identified metabolites were fitted by 157 a combination of a local baseline and Voigt functions based on the multiplicity of the NMR signal. GlycA 158 and GlycB signals were quantified by integrating, respectively, the areas between 2.005 and $2.054 \mathrm{ppm}$ and 159 between 2.086 and $2.054 \mathrm{ppm}$ above a local baseline aimed to remove the signal of the lipoproteins. Fitting 160 methods to quantify GlycA and GlycB signals were not used due to their heterogeneity and due to the 161 impossibility to completely distinguish them from the lipoprotein signal. The amide protein signals belong 162 to plasma proteins were quantified integrating the area between 6.000 and $10.000 \mathrm{ppm}$.

Plasma CRP quantification

164 The plasma concentration of CRP was determined using the Human C Reactive Protein ELISA kit (abcam, 165 ab99995). Patient plasma was diluted 1:80,000 in 1X Assay Diluent D.

166 Statistical and data analysis

167 Statistical analysis and graphical illustrations of the data were generated in the $\mathrm{R}$ (version 3.6.1) and $\mathrm{R}$ 168 studio (version 1.1.456) software using scripts developed in-house.

169 Wilcoxon Rank Sum test was used to compare differences in numerical covariates (e.g., age and metabolite

171 ethnicity). Spearman's test was used to calculate the correlation coefficient (rho) between variables. The

172 KODAMA algorithm was used to facilitate the identification of patterns representing underlying metabolic 173 phenotypes (metabotype) on all samples in the data set. Dendrograms were performed using the KODAMA 174 output and Ward linkage. Silhouette median value being used to evaluate the optimal number of clusters 175 with the number of possible clusters varying from 2 to 10 [32]. p-values less than 0.05 were considered to 176 be significant. To account for multiple testing, a false discovery rate (FDR) of $<10 \%$ was applied.

177 Discriminant analysis of metabolic profiles was performed using partial least-squares (PLS) analysis. To 178 assess the predictive ability of the PLS regression model, a 10-fold cross-validation was conducted as 
179 previously described [22]. The goodness of fit parameter (R2) and the predictive ability parameter (Q2) 180 were calculated using standard definitions [33].

\section{Results}

\section{South African patient cohort}

184 Although a few studies have been performed to investigate the metabolic alterations in the blood of patients 185 with PCa, high-risk populations are underrepresented and limited to AAs. In this study, we recruited 41 186 South African patients with PCa in order to generate a better understanding of the metabolic changes in the 187 unique South African setting. The majority of patients were characterized by a unique mixed ancestry $(61 \%)$ referred to as Coloured; the rest were self-classified as Black (22\%) and Whites (17\%). We classified the aggressiveness of cancer according to the National Comprehensive Cancer Network (NCCN) classification

190 (version 2.2020): i) very low, low, and intermediate risk; ii) high risk; and iii) very high risk. The clinical 191 and demographic features of the patients with PCa are reported in Table 1. Patients with regional or distant 192 metastasis were classified as a separate group. Patients that received androgen-deprivation therapy (ADT), 193 i.e., bilateral orchidectomy (BO), were considered as two distinct groups based on the evidence of 194 castration-resistant PCa (CRPC). In our cohort, we did not observe statistically significant difference among 195 ethnicity in term of NCCN classification in untreated patients, although, we reported an advanced clinical 196 stage in Black men with $71 \%$ of them classified as stage T3 or T4 compared to $19 \%$ of Coloured and $33 \%$ 197 of White men. This disparity was highlighted even by the PSA level. We reported extremely high values 198 of PSA (>100 ng/mL) in 57\% of Black compared to $9 \%$ of Coloured and $33 \%$ of White. As expected, we 199 observed a higher prevalence of diabetes and hypertension in the post-BO group.

200 GlycA and GlycB inflammatory biomarkers

201 Growing evidence implicates chronic inflammation as a contributor to PCa development and progression 202 to advanced metastatic disease [17], and as a driver of CRPC development in ADT [34, 35]. Recently, 
203 GlycA and GlycB have been identified as markers of systemic and chronic inflammation but their 204 association with PCa has not been described yet [36]. Here, we used the NMR spectroscopy to quantify the 205 signal associated with GlycA and GlycB and, for the first time, we investigated their association with the 206 aggressiveness of PCa. We noted that the values of both markers are higher in patients with very highly 207 aggressive PCa and metastatic PCa (Fig. 1a,e). Indeed, all patients whose GlycA and GlycB was higher 208 than the $80^{\text {th }}$ percentile were diagnosed with poorly differentiated PCa (i.e., Gleason score higher than or 209 equal to 8). Although the limited number of patients who had BO did not allow for enough statistical power, 210 we observed an increased value of both GlycA and GlycB in patients with CRPC (Fig. 1b,f).

211 Several studies have reported the association of GlycA and GlycB with key markers of cancer stratification,

212 such as CRP [37] (Fig. 1c,g). Here, we report for the first time a statistically significant correlation with 213 PSA (Fig. 1d,h). The correlation with PSA implies that a disparity in the GlycA and GlycB related to 214 ethnicity should be expected in patients with PCa. Noteworthy, the three highest values of GlycA and GlycB 215 were found in patients that identified themselves as Black.

216 Moreover, we quantified the amides of proteins from the NMR spectra. Albumin is the most concentrated 217 protein in the plasma and consequently, the protein amides concentration is highly related to the albumin

218 level. We reported a negative correlation between the NMR inflammatory marker GlycA (rho=-0.41; $219 P=0.00747)$ and $\mathrm{GlycB}($ rho $=-0.31 ; P=0.0462)$ with protein amides. Albumin is known to be a negative 220 APP that decreases in concentration during inflammation. This further supports our finding that the higher 221 values of GlycA and GlycB could be associated with inflammatory process in patients with PCa.

\section{Metabolic stratification of $P C a$}

223 Reprogramming of metabolism is a widely accepted hallmark of cancer development [21], however the 224 metabolic changes induced by inflammation in cancer patients have not been fully characterized.

225 Metabolomics represents an essential tool for the stratification of cancer patients into groups of patients 226 with similar metabolic profiles that could share the same clinicopathologic condition (e.g., systemic 
227 inflammation). Here, we quantified the metabolites from each plasma sample using the data collected by 228 the NMR experiments. In order to identify potential underlying metabolic phenotypes (a.k.a. metabotype) 229 in patients with treatment-naïve $\mathrm{PCa}$, we applied the unsupervised method KODAMA method to the 230 quantified metabolite concentrations (GlycA, GlycB, and protein amides were not considered in this 231 analysis). We identified four different metabotypes in the KODAMA score plot (Fig. 2a,b) using the 232 hierarchical clustering [38] on the KODAMA scores.

233 We clearly observed an association between the PCa aggressiveness (based on the NCCN classification) 234 and the metabotypes that we rank from I to IV in order of aggressiveness. When evaluating GlycB and 235 GlycA in the 4 metabotypes, we noted different levels of GlycB in each metabotypes with the highest levels 236 of both GlycA and GlycB in the metabotype IV. Clinical and demographic features of the metabotypes are 237 shown in Table 2.

238 The metabolic profile of the Metabotype IV is the most peculiar. Samples of the metabotype IV showed an 239 unprecedently well-defined fingerprint that may reflect a common biologic process that drives the 240 metabolic changes in the blood of patients with high GlycA and GlycB levels. The metabotype IV is formed 241 almost exclusively by patients categorized as very high risk and also includes a patient with metastatic PCa 242 (Table 2).

243 Noteworthy, a patient classified as low risk PCa based on the NCCN classification showed a metabolic 244 profile typical of Metabotype IV but with lower level of GlycA and GlycB. This patient died only 50 days 245 after sample collection due to pancreatic cancer. On the other hand, patients classified as very high 246 aggressiveness but that do not belong to the Metabotype IV showed a survival time longer than 3 years and 247 a lower Gleason score compared with patients belong to the Metabotype IV. We were unable to record the 248 date of death for 4 out of 7 patients belong to the Metabotype IV. These patients were lost to follow-up at 249 the hospital cancer center where they were recruited nor did they have any type of diagnostic test at a South 250 African clinic or hospital. Considering the severe condition of these patients and the absence of registered 251 diagnostic tests following the last visit, we assume the latter as a rough estimation of the time of survival. 
252 Almost all patients of the Metabotype IV seem to have died within one year after the sample collection 253 (Table 3).

254 Noteworthy, we reported a few clues of possible differences of the prostate tissue inflammation among the 255 metabotype. Of 14 patients in the Metabotype III, 3 patients had mild chronic inflammation and 2 patients 256 had chronic inflammation reported on the histological exam of their prostate tissue. Of the 10 patients of 257 the Metabotype II, 2 patients had mild chronic inflammation, 3 patients had acute-on-chronic inflammation, 258 and 1 had acute prostatitis on the histological exam.

\section{Metabolic profiling of PCa metabotypes}

260 The metabolic differences discriminating between among the four metabotypes appear to be clear (Fig. 2c). 261 An overview of some discriminative NMR signals among the four metabotypes are showed in Figure 3. 262 Although we were aware of the low number of patients in this cohort, we built a supervised PLS model to 263 evaluate the accuracy of the identification of the most aggressive metabotype (i.e., Metabotype IV) using 264 the metabolic profile. Using a double cross-validation approach, we calculated an accuracy value of $91.2 \%$, with a $95 \%$ coefficient interval of $86.0 \%-94.1 \%$. Next, we used the Wilcoxon rank-sum test to characterize this metabotype compared to the others (Supplementary Table 1). As previously mentioned, the Metabotype IV is characterized by higher values of the inflammatory markers GlycA $\left(P=7.06 \times 10^{-6}\right.$; FDR $\left.=7.24 \times 10^{-5}\right)$ and GlycB $\left(P=2.60 \times 10^{-6} ; \mathrm{FDR}=3.56 \times 10^{-5}\right)$ and lower protein level $\left(P=9.15 \times 10^{-5}\right.$; $\left.\mathrm{FDR}=5.36 \times 10^{-4}\right)$. In addition, we detected a higher level of mannose $\left(P=4.45 \times 10^{-3} ; \mathrm{FDR}=1.40 \times 10^{-2}\right)$, an important constituent of $\mathrm{N}$-glycans of glycoproteins [39]. Mannose residues in $\mathrm{N}$-glycans can be derived from either glycogen/glucose or mannose in the blood [39]. Moreover, we detected a reduced level of amino acids and their derivates in the Metabotype IV. Among them, the reduction of histidine is the most significant $\left(P=3.72 \times 10^{-7} ; \mathrm{FDR}=1.52 \times 10^{-5}\right)$. We noted an interesting clue of the possible role of microbiota in the inflammatory status of patients with PCa. We found desaminotyrosine, a metabolite produced by human enteric bacteria and able to induce type I interferon (IFN) response[40], prevalent in patients with very high aggressive PCa. 
277 Similar to the previous analysis, we also investigated the rearrangement of the lipoprotein profile using the 278 data from the application of the Bruker's B.I.-LISA protocols (Supplementary Table 2). The Bruker's B.I.-LISA protocols were used to characterize 114 parameters related to the lipoproteins, such as HDL,

281 lower level of apolipoprotein Apo-A1 $\left(P=2.67 \times 10^{-3} ; \mathrm{FDR}=6.10 \times 10^{-2}\right)$ and Apo-A2 $\left(P=4.04 \times 10^{-3}\right.$; $\left.282 \mathrm{FDR}=5.32 \times 10^{-2}\right)$, attributable to a reduced $\mathrm{HDL}$ particle number $\left(P=1.28 \times 10^{-2} ; \mathrm{FDR}=2.68 \times 10^{-1}\right)$, and higher 283 level of triglycerides in LDL of smaller size, including LDL-1 $\left(P=4.45 \times 10^{-3} ; \mathrm{FDR}=8.45 \times 10^{-2}\right)$ and LDL-2 $284\left(P=1.40 \times 10^{-3} ; \mathrm{FDR}=5.32 \times 10^{-2}\right)$. This finding completes the snapshot of the metabotype IV as a large 285 regulator of the blood constituents, including metabolites, proteins, and lipoproteins with several 286 implications for the role of inflammation as a confounding factor of PCa.

287 The levels of inflammatory NMR markers GlycA and GlycB have been shown to be highly correlated, as 288 previously reported in the literature [37]. In our study, we reported a Spearman's rank correlation rho of $2890.59\left(P=7.16 \times 10^{-5}\right)$. However, the biological meaning of the differences between GlycA and GlycB has not 290 yet been fully explored. In our cohort, we observed that two distinct metabotypes, Metabotype II and 291 Metabotype III, had a similar level of GlycA but different levels of GlycB $\left(P=1.42 \times 10^{-4}\right.$; FDR=6.46x10-4), 292 with the latter showing the higher level (Table S3). Moreover, Metabotype III seems to be associated with 293 extremely reduced levels of lipids (Fig. 3a,e). We discovered a deep difference in the lipoprotein profile 294 between Metabotype III and Metabotype II using the B.I.-LISA protocols (Supplementary Table 4). 295 Figure 4 shows a graphical representation of the lipoprotein profile changes among metabotypes. Besides 296 the evident reduction of VLDL, we also noted the lower values of Apo-A1 and Apo-A2 that could help to 297 characterize the differences between GlycA and GlycB.

\section{Discussion}


300 PCa shows significant ethnic disparity among men with African ancestry representing a well-established 301 risk factor. In a recent study, all patients who underwent a prostate biopsy from July 2008 to July 2014 in 302 one of the hospitals included in our cohort (Groote Schuur Hospital, Cape Town) were recorded [41]. 303 Among all patients diagnosed with $\mathrm{PCa}, 41 \%$ and $21 \%$ were classified as high and very high risk PCa 304 (NCCN classification), respectively. Although the percentage of clinically advanced cases is already 305 impressive if compared to American or European studies, the relative percentage of cases with very high aggressive $\mathrm{PCa}$ is even higher (33\%) if we consider only the Black population.

In this first metabolomic study of PCa conducted on an African population, we profiled the plasma samples of men of different ancestry. We observed in men with very high aggressive PCa higher levels of the NMR inflammatory markers GlycA and GlycB, probably due to the increased concentration of positive APPs and the complexity of their glycan structures. Moreover, we noted a simultaneous reduction of the signal from protein source likely attributable to a reduction of the albumin level, a negative APP.

Interestingly, in our cohort we discovered four distinct metabotypes associated with the aggressiveness of $\mathrm{PCa}$, each one characterized by a unique metabolic fingerprint. A metabotype identified as a subgroup of patients with very high aggressive PCa (that we named Metabotype IV) was characterized by the highest values of GlycA and GlycB and by deep changes of the plasma metabolome. We observed a lower level of histidine that could reinforce our hypothesis of inflammatory processes underlying the Metabotype IV. Indeed, histidine has been already associated with inflammatory processes and in particular, it has been negatively correlated with other inflammatory markers, such as IL-6 and CRP [42]. In addition, it has been reported that inflammation may alter the lipoprotein profile as well, for example modulating the HDL functions [43-45] as we observed. Recently, it has been shown that high levels of triglycerides and glucose and low levels of HDL cholesterol and Apo-A1 are related to increased PCa risk and its severity [46]. Moreover, low HDL was reported to be a risk and prognostic factor for PCa in several epidemiologic studies [47]. Elevated serum triglycerides were associated with an increased risk of PCa recurrence [48]. Lower levels of Apo-A1 and Apo-A2, and a higher level of triglycerides in LDL, reported in this study, are 
consistent with these processes and we suggest that inflammation could be a driving factor of the lipoprotein profile changes observed in Metabotype IV.

327 Although in vitro studies reported that aerobic glycolysis can be directly induced by an inflammatory microenvironment $[49,50]$, we did not observe any metabolic signature of the Warburg effect in the plasma of the patients with an inflammatory status associated to the PCa. Indeed, plasma metabolome do not necessarily reflect the Warburg effect present in the tumor tissue. As reported in a study on metastatic

331 colorectal cancer (CRC) [22] where the Warburg effect is well-established, patients with mCRC showed

332 lower serum levels of lactate and non-statistically significant changes of glucose compared to the controls.

333 Liver gluconeogenesis [51] and insulin resistance associated with inflammation [52] may mask anaerobic 334 dissimilation of glucose and lactate production.

335 Furthermore, we highlighted two other distinct metabotypes characterized by large differences in the 336 lipoprotein profile. We noted a higher concentration of GlycB in the Metabotype III, which is characterized

337 by a higher number of patients with very high aggressive PCa compared to the Metabotype II. No differences in the concentration of GlycA and higher levels of GlycB could be due to an elevated sialylation post-translational modification on glycosylated proteins. Complex biantennary glycoforms with $\alpha 2,3$-sialic acid have been associated with aggressive PCa [53-55]. Here, for the first time, we reported the association of lower level of the inflammatory NMR biomarker GlycB with a higher concentration of VLDL. Since the concentration of dietary intake can modulate the VLDL, this finding will further enrich the long-standing debate over the role of dietary fat in promoting PCa [56, 57].

In men diagnosed with $\mathrm{PCa}$, the selection of the treatment, including the type of therapy and its aggressiveness, is often based on patient age and life expectancy. In an era of precision medicine, an estimate of the threat of disease and the benefit and the costs of intervention within the context of the patient's characteristics and desires should be taken into consideration regarding the decision of the treatment. In this study, we identified a set of patients with very high aggressive PCa with extremely reduced survival time. These patients, belonging to the Metabotype IV, are characterized by a similar 
metabolic profile predictable with high accuracy. Our results postulate that this subgroup may be most

351 likely to benefit from combination therapy that associates the androgen deprivation in conjunction with 352 drugs aiming to reduce the level of systemic inflammation.

353 In this context, metabolomics could represent an invaluable tool for the stratification of patients with very

354 high aggressive PCa. The life expectancy difference highlights the need to consider an appropriate medical 355 treatment for patients within Metabotype IV. We hypothesize that these patients could largely benefit from 356 daily treatment with corticosteroids to reduce the systemic inflammation improving the overall survival, 357 along with the need for subsequent therapy. However, the only clinical settings of corticosteroids treatment 358 with proved clinical utility in PCa treatment is in combination with abiraterone. We consider the lack of 359 clinical investigation for almost all patients of the presence of distant metastasis as a limitation of this study.

360 Considering the clinical and histopathological features, we are aware that PCa could have spread to distant 361 organs in the patients with very high risk PCa belonging to the Metabotype IV. The current project is still 362 ongoing and we are recruiting more patients with PCa to further validate our results in a larger cohort.

\section{Conclusions}

364 Integrating a metabolomic analysis as a tool for patient stratification could be important to identify those 365 patients with very high risk PCa and short life expectancy, as they may benefit from therapeutic 366 interventions, targeting the lowering of systemic inflammation. Thus, further studies are necessary to better 367 characterize this group of patients and determine the costs and benefits of corticosteroid treatment in terms 368 of survival time and quality of life.

369 In conclusion, this study answers the urgent need for new precision medicine approaches aiming to profile 370 aggressive and lethal PCa in men of African ancestry. New non-invasive metabolite biomarkers are 371 necessary to improve the treatment decision, which will improve therapeutic outcomes in African patients. 


\section{Disclosure of Potential Conflicts of Interest}

375 No potential conflicts of interest were disclosed.

\section{Funding}

378 Support by The International Centre for Genetic Engineering and Biotechnology, ICGEB (LFZ); ICGEB 379 Arturo Falaschi fellowship (SC); Joint Research Grant South Africa/Switzerland Research Partnership 380 Programme Bilateral Agreement (LFZ, CVC, GMC); The South African National Research Foundation 381 (NRF) for Professional Development Programme (MW); NIH/NCI P30 CA006516 (TAL)

\section{Contributions}

384 L.F.Z. conceived the project and designed experiments; S.C., M.W., and C.L. performed the experiments 385 and analysis of the data; S.C. wrote the paper with assistance and feedback of all the other co-authors. In 386 addition, all authors participated in revising the work critically for important intellectual content and 387 approved the final version. 
Table 1. Clinical demographics of PCa patients

\begin{tabular}{|c|c|c|c|c|c|c|c|c|}
\hline \multirow[b]{2}{*}{ Feature } & \multicolumn{5}{|c|}{ Treatment-naïve } & \multicolumn{3}{|c|}{ post-BO } \\
\hline & $\begin{array}{c}\text { intermediate, } \\
\text { low and very } \\
\text { low }(n=14)\end{array}$ & $\begin{array}{l}\text { high } \\
(n=7)\end{array}$ & $\begin{array}{l}\text { very high } \\
(n=12)\end{array}$ & $\begin{array}{l}\text { metastatic } \\
\qquad(n=1)\end{array}$ & $\begin{array}{l}\text { Total } \\
n=34)\end{array}$ & $\begin{array}{c}\text { non-CRPC } \\
(n=4)\end{array}$ & $\begin{array}{l}\text { CRPC } \\
(n=3)\end{array}$ & $\begin{array}{l}\text { Total } \\
(n=7)\end{array}$ \\
\hline Age (year), median $[95 \% \mathrm{Cl}]$ & $65[5677]$ & 70 [52 90] & $64[5786]$ & 75 [75 75] & $68[6374]$ & $72[6373]$ & $66[6470]$ & $70\left[\begin{array}{lll}65 & 72\end{array}\right]$ \\
\hline \multicolumn{9}{|l|}{ Ancestry, $\mathrm{n}(\%)$} \\
\hline Black & $1(7.1)$ & $1(14.3)$ & $4(33.3)$ & $1(100.0)$ & $7(20.6)$ & $1(25.0)$ & $1(33.3)$ & $2(28.6)$ \\
\hline Coloured & $10(71.4)$ & $5(71.4)$ & $6(50.0)$ & $0(0.0)$ & $21(61.8)$ & $2(50.0)$ & $1(33.3)$ & $3(42.8)$ \\
\hline Coloured/Black & $0(0.0)$ & $0(0.0)$ & $0(0.0)$ & $0(0.0)$ & $0(0.0)$ & $1(25.0)$ & $0(0.0)$ & $1(14.3)$ \\
\hline White & $3(21.4)$ & $1(14.3)$ & $2(16.7)$ & $0(0.0)$ & $6(17.6)$ & $0(0.0)$ & $1(33.3)$ & $1(14.3)$ \\
\hline PSA (ng/mL), median [95\%Cl] & 9 [3 19] & 23 [17 31] & 138 [29 3919] & $>5000$ & $21[1284]$ & $4[240]$ & 332 [49 1128] & 34 [4 188] \\
\hline \multicolumn{9}{|l|}{ Diabetes, n (\%) } \\
\hline no & $10(71.4)$ & $5(71.4)$ & $12(100.0)$ & $1(100.0)$ & $28(82.4)$ & $3(75.0)$ & $2(66.7)$ & $5(71.4)$ \\
\hline yes & $4(28.6)$ & $2(28.6)$ & $0(0.0)$ & $0(0.0)$ & $6(17.6)$ & $1(25.0)$ & $1(33.3)$ & $2(28.6)$ \\
\hline \multicolumn{9}{|l|}{ Hypertension, n (\%) } \\
\hline no & $7(50.0)$ & $5(71.4)$ & $10(83.3)$ & $1(100.0)$ & $23(67.6)$ & $3(75.0)$ & $1(33.3)$ & $4(57.1)$ \\
\hline yes & $7(50.0)$ & $2(28.6)$ & $2(16.7)$ & $0(0.0)$ & $11(32.4)$ & $1(25.0)$ & $2(66.7)$ & $3(42.9)$ \\
\hline \multicolumn{9}{|l|}{ Smoker, n (\%) } \\
\hline no & $10(71.4)$ & $6(85.7)$ & $9(75.0)$ & $1(100.0)$ & $26(76.5)$ & $4(100.0)$ & $1(33.3)$ & $5(71.4)$ \\
\hline yes & $4(28.6)$ & $1(14.3)$ & $3(25.0)$ & $0(0.0)$ & $8(23.5)$ & $0(0.0)$ & $2(66.7)$ & $2(28.6)$ \\
\hline
\end{tabular}


392 Table 2. Clinical and demographic features of the metabotypes identified thought KODAMA analysis.

393

\begin{tabular}{|c|c|c|c|c|}
\hline Feature & Metabotype I & Metabotype II & Metabotype III & Metabotype IV \\
\hline \multicolumn{5}{|c|}{ NCCN classification, $\mathrm{n}(\%)$} \\
\hline very low & $1(33.3)$ & $0(0.0)$ & $1(7.1)$ & $0(0.0)$ \\
\hline low & $0(0.0)$ & $1(10.0)$ & $1(7.1)$ & $1(14.3)$ \\
\hline intermediate & $2(66.7)$ & $4(40.0)$ & $3(21.4)$ & $0(0.0)$ \\
\hline high & $0(0.0)$ & $3(30.0)$ & $4(28.5)$ & $0(0.0)$ \\
\hline very high & $0(0.0)$ & $2(20.0)$ & $5(35.7)$ & $5(71.4)$ \\
\hline metastatic & $0(0.0)$ & $0(0.0)$ & $0(0.0)$ & $1(14.3)$ \\
\hline \multicolumn{5}{|l|}{ Gleason Score, n (\%) } \\
\hline $3+3$ & $1(33.3)$ & $1(10.0)$ & $5(35.7)$ & $1(14.3)$ \\
\hline $3+4$ & $1(33.3)$ & $4(40.0)$ & 6 (42.9) & $0(0.0)$ \\
\hline $4+3$ & $1(33.3)$ & $1(10.0)$ & $0(0.0)$ & $0(0.0)$ \\
\hline $3+5$ & $0(0.0)$ & $0(0.0)$ & $0(0.0)$ & $1(14.3)$ \\
\hline $4+5$ & $0(0.0)$ & $4(40.0)$ & $3(21.4)$ & $0(0.0)$ \\
\hline $5+4$ & $0(0.0)$ & $0(0.0)$ & $0(0.0)$ & $2(28.6)$ \\
\hline $5+5$ & $0(0.0)$ & $0(0.0)$ & $0(0.0)$ & $3(42.9)$ \\
\hline Age, median $[95 \% \mathrm{Cl}]$ & $71[6474]$ & 68 [56 90] & $65[5185]$ & $74[5978]$ \\
\hline \multicolumn{5}{|l|}{ Ancestry, $\mathrm{n}(\%)$} \\
\hline Black & $1(33.3)$ & $1(10.0)$ & $2(14.3)$ & $3(42.9)$ \\
\hline Mixed ancestry & $1(33.3)$ & $7(70.0)$ & $11(78.6)$ & $2(28.6)$ \\
\hline White & $1(33.3)$ & $2(20.0)$ & $1(7.1)$ & $2(28.6)$ \\
\hline PSA, median $[95 \% \mathrm{Cl}]$ & 9 [3 9] & 18 [5 233] & $25\left[\begin{array}{ll}5 & 126\end{array}\right]$ & 738 [26 5000] \\
\hline \multicolumn{5}{|l|}{ Diabetes, n (\%) } \\
\hline no & $1(33.3)$ & $7(70.0)$ & 13 (92.9) & $7(100.0)$ \\
\hline yes & $2(66.7)$ & $3(30.0)$ & $1(7.1)$ & $0(0.0)$ \\
\hline \multicolumn{5}{|l|}{ Hypertension, n (\%) } \\
\hline no & $0(0.0)$ & $8(80.0)$ & $9(64.3)$ & $6(85.7)$ \\
\hline yes & $3(100.0)$ & $2(20.0)$ & $5(35.7)$ & $1(14.3)$ \\
\hline \multicolumn{5}{|l|}{ Smoker, n (\%) } \\
\hline no & $2(66.7)$ & $8(80.0)$ & $9(64.3)$ & $7(100.0)$ \\
\hline yes & $1(33.3)$ & $2(20.0)$ & $5(35.7)$ & $0(0.0)$ \\
\hline
\end{tabular}


396 Table 3. Demographics and clinical features of patients belonging to the Metabotype IV and patients

397 classified as very high aggressiveness that do not belong to the Metabotype IV.

398

\begin{tabular}{|c|c|c|c|c|c|c|c|c|c|c|c|c|c|}
\hline 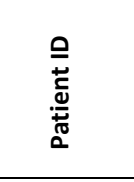 & 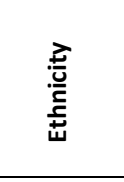 & 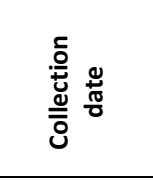 & 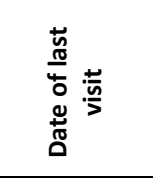 & $\begin{array}{l}\frac{5}{0} \\
0 \\
0 \\
0 \\
0 \\
0 \\
0 \\
0 \\
0 \\
0\end{array}$ & 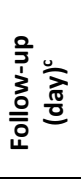 & 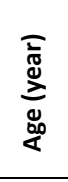 & $\frac{\vec{a}}{\frac{\vec{\varepsilon}}{50}}$ & 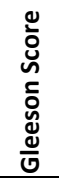 & 容 & z⿺辶寸 & 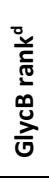 & $\begin{array}{l}0 \\
\sum_{2}^{0} \\
0 \\
\frac{0}{\pi} \\
\stackrel{0}{0} \\
\sum\end{array}$ & 壱 \\
\hline SAPC0159 & Black & 2017/03/01 & $2017 / 03 / 23$ & & 22 & 75.2 & $>5000$ & $5+5$ & T3/T4 & metastatic & 3 & IV & \\
\hline SAPC0090 & Coloured & $2015 / 05 / 08$ & & $2015 / 06 / 27$ & 50 & 78.2 & 6.3 & $3+3$ & $\mathrm{~T} 1 \mathrm{c}$ & low & 8 & IV & a \\
\hline SAPC0192 & Coloured & $2017 / 07 / 31$ & & $2017 / 12 / 19$ & 141 & 80.9 & 41.68 & $4+5$ & $\mathrm{~T} 2 \mathrm{a}$ & very high & 7 & III & $b$ \\
\hline SAPC0080 & Black & $2014 / 10 / 31$ & $2015 / 04 / 21$ & & 172 & 77.8 & 738 & $3+5$ & $\mathrm{~T} 4$ & very high & 2 & IV & \\
\hline SAPC0180 & Black & $2017 / 06 / 23$ & $2017 / 12 / 12$ & & 172 & 58.1 & $>5000$ & $5+5$ & T3/T4 & very high & 1 & IV & \\
\hline SAPC0249 & Coloured & $2018 / 03 / 25$ & 2019/01/07 & & 288 & 63 & 1070 & $5+4$ & T3 & very high & 4 & IV & \\
\hline SAPC0078 & White & 2014/11/07 & & 2015/10/31 & 358 & 74.3 & 135.79 & $5+5$ & $\mathrm{~T} 4$ & very high & 5 & IV & \\
\hline SAPC0193 & Coloured & $2017 / 08 / 11$ & 2019/04/26 & & 623 & 63.5 & 48.85 & $3+4$ & T3 & very high & 9 & III & \\
\hline SAPC0070 & Coloured & $2014 / 01 / 08$ & & 2015/12/11 & 702 & 65.4 & 34.8 & $4+5$ & $\mathrm{~T} 2 \mathrm{~b}$ & very high & 13 & II & \\
\hline SAPC0191 & White & $2017 / 07 / 28$ & & 2019/08/03 & 736 & 61.8 & 576 & $5+4$ & T3 & very high & 6 & IV & \\
\hline SAPC0195 & Black & $2017 / 08 / 13$ & 2020/01/30 & & 900 & 87.2 & 26.53 & $4+5$ & T3 & very high & 11 & III & \\
\hline SAPC0120 & Black & 2016/10/03 & 2019/09/30 & & 1092 & 82.3 & 289.9 & $4+5$ & T3 & very high & 14 & II & \\
\hline SAPC0108 & Coloured & $2016 / 06 / 27$ & & $2020 / 04 / 17$ & 1390 & 56.6 & 96.3 & $3+4$ & T4 & very high & 12 & III & \\
\hline SAPC0076 & Coloured & $2014 / 09 / 26$ & 2020/06/04 & & 2078 & 63.5 & 140.42 & $3+4$ & T3/T4 & very high & 10 & III & \\
\hline
\end{tabular}

$400{ }^{a}$ The patient was diagnosed with pancreas cancer 19 day after sample collection.

$401 \mathrm{~b}$ The patient died for bone metastasis 5 months after sample collection.

$402{ }^{\mathrm{c}}$ Number of days between the date of collection and the date of last visit at hospital or the date of death (if 403 recorded).

$404{ }^{\mathrm{d}}$ This number represent the rank of order of the GlycB values starting from the highest value. 
Figure 1. GlycA concentration in (a) treatment-naïve and (b) post-treatment. (c) Correlation between 407 GlycA and CRP. (d) Correlation between GlycA and PSA. GlycB concentration in (e) treatment-naïve and
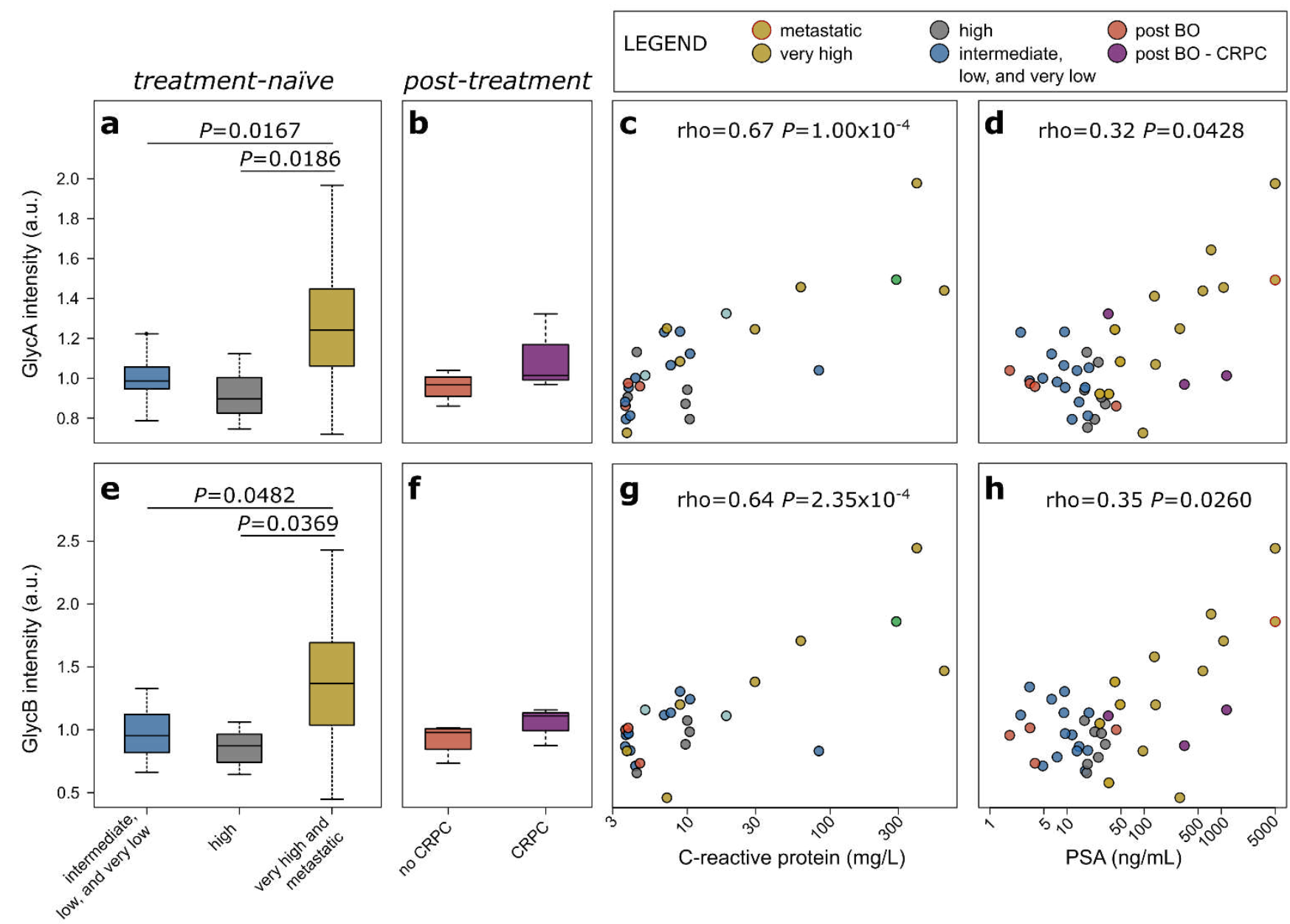
412 Figure 2. KODAMA score plot of plasma PCa samples colored according to NCCN classification. The

413 size is proportional to (a) the GlycA intensity and (b) the GlycB intensity. (c) Heatmap of the metabolic 414 profiles.
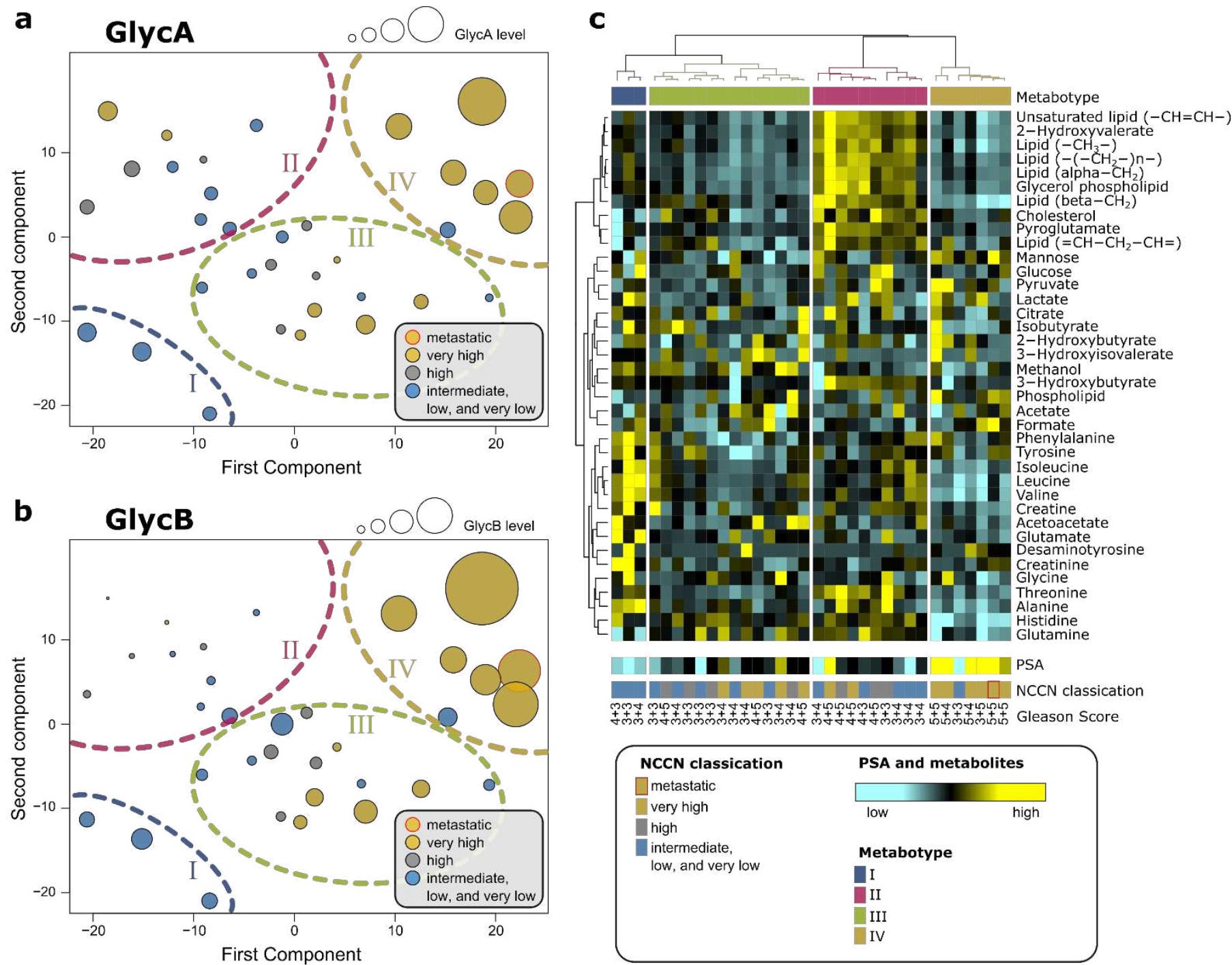

\begin{tabular}{|l|l|}
\hline \multicolumn{1}{|c|}{ NCCN classication } & PSA and metabolites \\
$\begin{array}{l}\text { metastatic } \\
\text { very high } \\
\text { high } \\
\text { intermediate, } \\
\text { low, and very low }\end{array}$ & low high \\
& Metabotype \\
& I \\
& II \\
& III \\
& IV \\
\hline
\end{tabular}


418 Figure 3. (a) NMR profiles of the plasma of three different spectral regions and Box-whiskers plots of the 419 intensity of (b) GlycA and (c) GlycB, the concentration of (d) histidine and the number of (e) VLDL 420 particles across the four metabotypes.

\section{a}

NCCN classification

$\square$ metastatic
$\square$ very high
high
$\square$ intermediate,
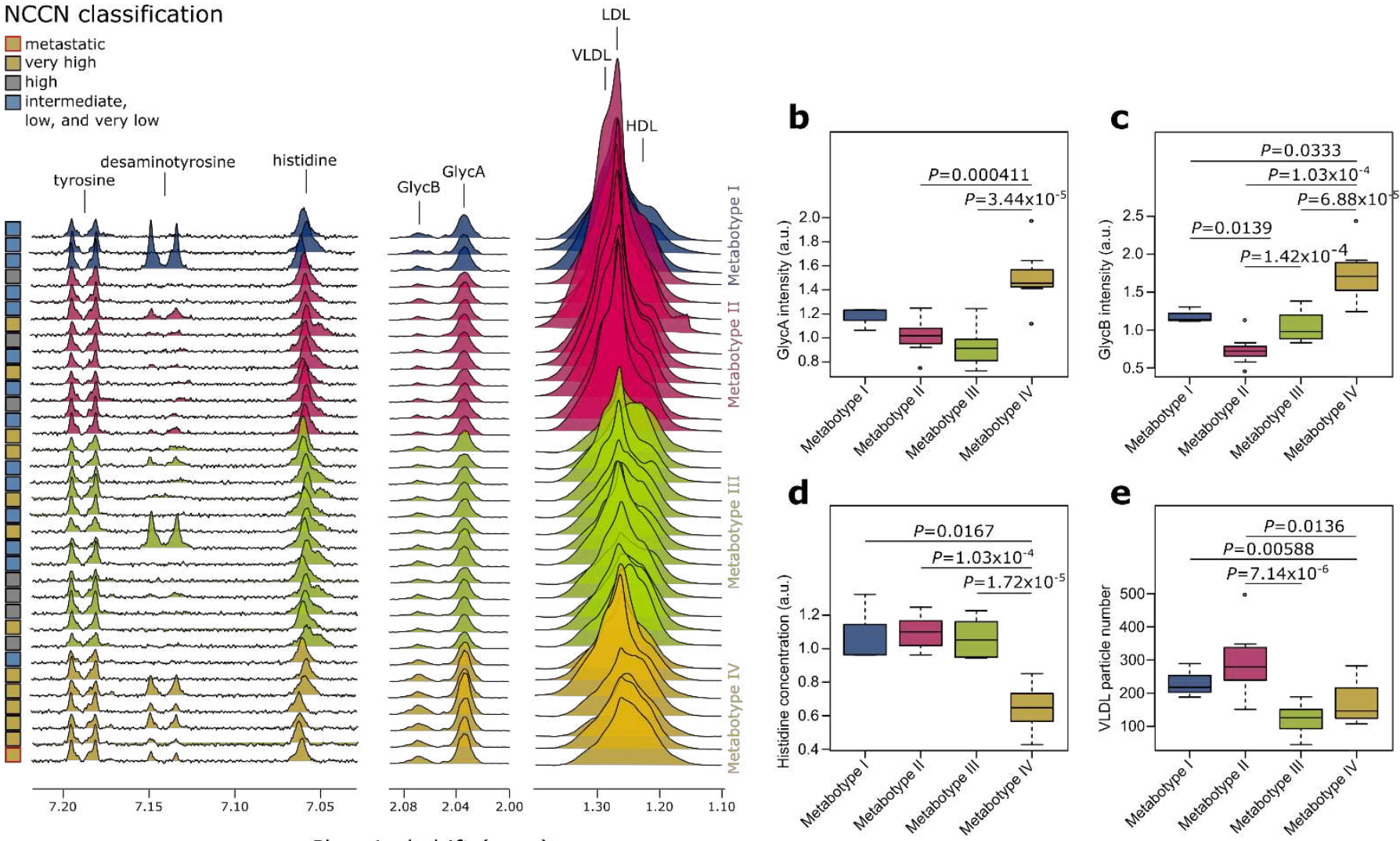
Figure 4. Graphics illustration of the changes of the lipoprotein profile.

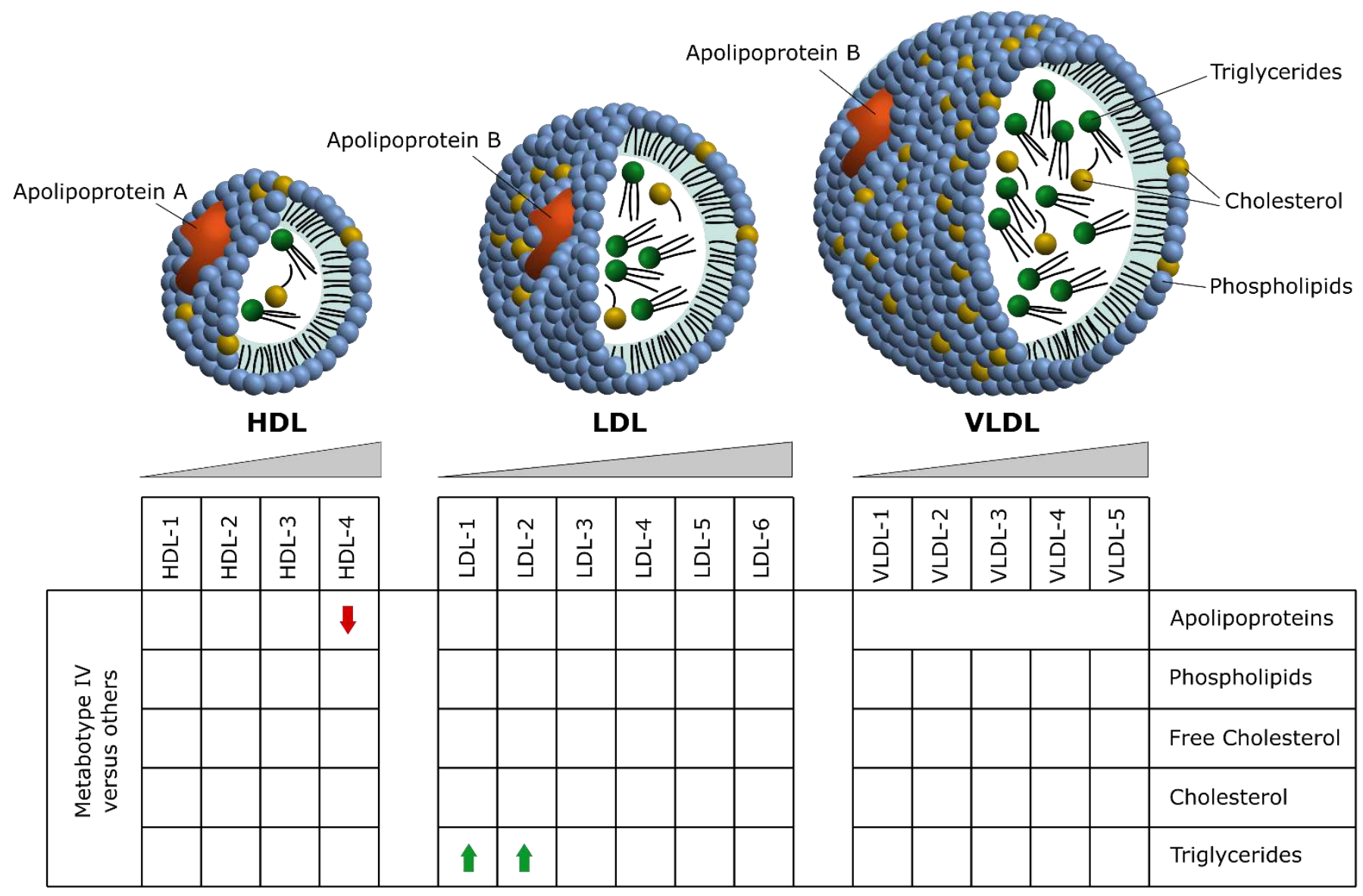

\begin{tabular}{|c|c|c|c|c|c|c|c|c|c|c|c|c|c|}
\hline \multirow{5}{*}{ 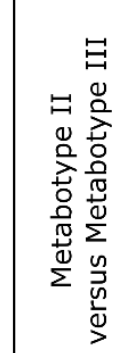 } & & $\boldsymbol{1}$ & - & & & $\downarrow$ & $\boldsymbol{1} \boldsymbol{t}$ & \multicolumn{5}{|c|}{ Ate } & Apolipoproteins \\
\hline & & & & & & & $\boldsymbol{1} \boldsymbol{t}$ & Atê & Atê & 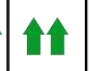 & At & 1t & Phospholipids \\
\hline & $\nabla$ & & & $\nabla$ & & & $\boldsymbol{1}$ & Atê & Â & $\hat{1} \boldsymbol{1} \hat{t}$ & 令 & t1 & Free Cholesterol \\
\hline & & & & & & & t1 & Atê & 11 & tA & At & $\boldsymbol{1}$ & Cholesterol \\
\hline & & 1 & t1 & & $\sqrt{ }$ & & $\boldsymbol{1}$ & Atê & Atê & 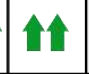 & At & $\boldsymbol{1}$ & Triglycerides \\
\hline
\end{tabular}

Lower:

$0.001<p$-value $<0.01$

Higher:

$$
\begin{aligned}
& \text { Af } 0.0001<p \text {-value } \leq 0.001 \\
& \text { Af } 0.00001<p \text {-value } \leq 0.0001
\end{aligned}
$$


429 Table S1. Statistical comparison between the metabolic profiles of Metabotype IV versus the others.

\begin{tabular}{|c|c|c|c|c|c|}
\hline Feature & Metabotype IV, median [IQR] & Others, median [IQR] & log change & p-value & FDR \\
\hline Histidine & $0.647[0.5660 .732]$ & $1.067[0.9811 .163]$ & -0.74 & $3.72 \times 10^{-7}$ & $1.52 \times 10^{-5}$ \\
\hline Lipid (beta-CH2) & $0.743[0.60 .785]$ & 1.12 [0.989 1.239] & -0.69 & $7.44 \times 10^{-7}$ & $1.52 \times 10^{-5}$ \\
\hline GlycB & $1.706[1.5241 .89]$ & $0.959[0.7821 .126]$ & 0.89 & $2.60 \times 10^{-6}$ & $3.56 \times 10^{-5}$ \\
\hline GlycA & $1.455\left[\begin{array}{lll}1.425 & 1.568]\end{array}\right.$ & $0.981[0.8921 .075]$ & 0.61 & $7.06 \times 10^{-6}$ & $7.24 \times 10^{-5}$ \\
\hline Valine & $0.62[0.5280 .668]$ & $1.002[0.9151 .244]$ & -0.77 & $6.73 \times 10^{-5}$ & $4.60 \times 10^{-4}$ \\
\hline Leucine & $0.624\left[\begin{array}{llll}0.487 & 0.764\end{array}\right]$ & $1.065[0.8781 .282]$ & -0.81 & $6.73 \times 10^{-5}$ & $4.60 \times 10^{-4}$ \\
\hline Protein & $0.9[0.870 .939]$ & $1.021[0.9791 .071]$ & -0.19 & $9.15 \times 10^{-5}$ & $5.36 \times 10^{-4}$ \\
\hline Alanine & $0.706\left[\begin{array}{lll}0.578 & 0.774\end{array}\right]$ & $1.075[0.891 .234]$ & -0.69 & $2.10 \times 10^{-4}$ & $1.07 \times 10^{-3}$ \\
\hline Isoleucine & $0.616[0.5480 .811]$ & $1.076[0.8961 .224]$ & -0.78 & $3.45 \times 10^{-4}$ & $1.57 \times 10^{-3}$ \\
\hline Glutamine & $0.842[0.7470 .976]$ & 1.098 [1.007 1.203] & -0.4 & $1.54 \times 10^{-3}$ & $6.33 \times 10^{-3}$ \\
\hline Lipid (-CH3-) & $0.868[0.8260 .946]$ & $1.062[0.9481 .189]$ & -0.28 & $2.67 \times 10^{-3}$ & $9.97 \times 10^{-3}$ \\
\hline Mannose & 1.368 [1.212 1.497$]$ & $1.011[0.7541 .173]$ & 0.46 & $4.45 \times 10^{-3}$ & $1.40 \times 10^{-2}$ \\
\hline Threonine & $0.663[0.540 .829]$ & $1.042[0.7831 .49]$ & -0.78 & $4.45 \times 10^{-3}$ & $1.40 \times 10^{-2}$ \\
\hline 2-Hydroxyvalerate & $0.867\left[\begin{array}{lll}0.853 & 0.938\end{array}\right]$ & $1.053[0.9611 .222]$ & -0.3 & $5.22 \times 10^{-3}$ & $1.53 \times 10^{-2}$ \\
\hline 3-Hydroxybutyrate & 0.875 [0.812 0.899] & $1.029[0.9651 .196]$ & -0.42 & $6.11 \times 10^{-3}$ & $1.67 \times 10^{-2}$ \\
\hline Unsaturated lipid $(-\mathrm{CH}=\mathrm{CH}-)$ & $0.769\left[\begin{array}{lll}0.723 & 0.922\end{array}\right]$ & 1.05 [0.904 1.332] & -0.46 & $8.27 \times 10^{-3}$ & $1.99 \times 10^{-2}$ \\
\hline Glycorol phospholipid & $0.475\left[\begin{array}{lll}0.276 & 0.577\end{array}\right]$ & $1.24[0.5521 .922]$ & -1.39 & $8.27 \times 10^{-3}$ & $1.99 \times 10^{-2}$ \\
\hline Creatine & $0.493\left[\begin{array}{lll}0.362 & 0.765]\end{array}\right.$ & $1.103[0.637$ 1.575] & -1.08 & $1.10 \times 10^{-2}$ & $2.51 \times 10^{-2}$ \\
\hline Pyroglutamate & $0.879[0.8640 .951]$ & $1.023[0.961 .239]$ & -0.25 & $1.45 \times 10^{-2}$ & $2.98 \times 10^{-2}$ \\
\hline Phospholipid & $1.184[1.11 .311]$ & $1.014[0.9261 .118]$ & 0.23 & $1.45 \times 10^{-2}$ & $2.98 \times 10^{-2}$ \\
\hline Desaminotyrosine & $13.361[6.03619 .946]$ & $1\left[\begin{array}{ll}1 & 1\end{array}\right]$ & 0.79 & $2.11 \times 10^{-2}$ & $4.12 \times 10^{-2}$ \\
\hline Isobutyrate & $0.643[0.5880 .868]$ & $0.959[0.8481 .202]$ & -0.35 & $3.89 \times 10^{-2}$ & $7.25 \times 10^{-2}$ \\
\hline Glutamate & $0.702\left[\begin{array}{llll}0.467 & 0.938\end{array}\right]$ & $1.146[0.6641 .378]$ & -0.87 & $4.85 \times 10^{-2}$ & $8.64 \times 10^{-2}$ \\
\hline Pyruvate & $1.781[1.1312 .386]$ & $1\left[\begin{array}{lll}0.643 & 1.507]\end{array}\right.$ & 0.74 & $8.87 \times 10^{-2}$ & $1.52 \times 10^{-1}$ \\
\hline Lipid (-(-CH2-)n-) & $0.825[0.7730 .992]$ & $1.051[0.8921 .512]$ & -0.43 & $9.74 \times 10^{-2}$ & $1.60 \times 10^{-1}$ \\
\hline Lipid (alpha-CH2) & $0.518\left[\begin{array}{lll}0.491 & 0.974\end{array}\right]$ & $0.943[0.6711 .98]$ & -0.86 & $1.17 \times 10^{-1}$ & $1.84 \times 10^{-1}$ \\
\hline Methanol & 0.919 [0.682 1.255$]$ & $1.244[0.9241 .534]$ & -0.32 & $1.39 \times 10^{-1}$ & $2.10 \times 10^{-1}$ \\
\hline Acetoacetate & $0.833[0.6430 .94]$ & $0.958[0.7341 .286]$ & -0.52 & $1.77 \times 10^{-1}$ & $2.59 \times 10^{-1}$ \\
\hline Formate & 1.119 [0.951 1.352$]$ & $1.023[0.7671 .158]$ & 0.24 & $2.57 \times 10^{-1}$ & $3.63 \times 10^{-1}$ \\
\hline 2-Hydroxybutyrate & $0.989[0.8541 .332]$ & $0.933[0.7591 .196]$ & 0.21 & $2.75 \times 10^{-1}$ & $3.76 \times 10^{-1}$ \\
\hline 3-Hydroxyisovalerate & $0.484\left[\begin{array}{lll}0.392 & 2.729\end{array}\right]$ & $0.663[0.4893 .622]$ & -0.03 & $3.35 \times 10^{-1}$ & $4.29 \times 10^{-1}$ \\
\hline Lipid (=CH-CH2-CH=) & 0.95 [0.819 1.004] & 1.05 [0.86 1.102] & -0.1 & $3.35 \times 10^{-1}$ & $4.29 \times 10^{-1}$ \\
\hline Glycine & $1.003\left[\begin{array}{lll}0.886 & 1.145]\end{array}\right.$ & 1.109 [0.979 1.193] & -0.14 & $4.27 \times 10^{-1}$ & $5.30 \times 10^{-1}$ \\
\hline Cholesterol backbone & $1.047[0.9121 .146]$ & 1.131 [0.93 1.324] & -0.11 & $5.04 \times 10^{-1}$ & $6.08 \times 10^{-1}$ \\
\hline Glucose & 1.015 [0.865 1.093] & 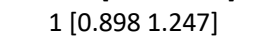 & -0.13 & $5.59 \times 10^{-1}$ & $6.51 \times 10^{-1}$ \\
\hline Lactate & $1.388[0.7381 .634]$ & 0.869 [0.698 1.334$]$ & 0.27 & $5.88 \times 10^{-1}$ & $6.51 \times 10^{-1}$ \\
\hline Citrate & 0.969 [0.8 1.195] & $1.042[0.8051 .285]$ & -0.1 & $5.88 \times 10^{-1}$ & $6.51 \times 10^{-1}$ \\
\hline Creatinine & $0.974[0.8731 .149]$ & $1.004[0.8071 .14]$ & -0.03 & $7.39 \times 10^{-1}$ & $7.97 \times 10^{-1}$ \\
\hline Phenylalanine & $0.958[0.8211 .113]$ & $0.95[0.7991 .111]$ & 0.03 & $8.03 \times 10^{-1}$ & $8.44 \times 10^{-1}$ \\
\hline Acetate & $1.053[0.8731 .247]$ & 1.004 [0.902 1.255] & -0.05 & $9.01 \times 10^{-1}$ & $9.23 \times 10^{-1}$ \\
\hline Tyrosine & 1.077 [0.87 1.186] & $1.043[0.8651 .148]$ & 0.01 & 1.00 & 1.00 \\
\hline
\end{tabular}


434 Table S2. Statistical comparison between the lipoprotein profiles of Metabotype IV versus the others.

\begin{tabular}{|c|c|c|c|c|c|}
\hline Feature & Metabotype IV, median [IQR] & Others, median [IQR] & log change & p-value & FDR \\
\hline Main_Parameters_Apo.A2 & $25.01[22.86526 .685]$ & $30.93[27.9733 .845]$ & -0.32 & $1.04 \times 10^{-3}$ & $5.32 \times 10^{-2}$ \\
\hline HDL_Subfractions_Apo.A2_HDL.4 & 12.15 [11.055 13.345] & 18.24 [14.905 21.8] & -0.6 & $1.04 \times 10^{-3}$ & $5.32 \times 10^{-2}$ \\
\hline LDL_Subfractions_Triglycerides_LDL.2 & $3.59[3.33$ 3.905] & 2.08 [1.825 2.755] & 0.63 & $1.40 \times 10^{-3}$ & $5.32 \times 10^{-2}$ \\
\hline MainFractions_Apo.A2_HDL & 26.37 [24.41 28.245] & 31.88 [29.105 34.71] & -0.31 & $1.87 \times 10^{-3}$ & $5.34 \times 10^{-2}$ \\
\hline HDL_Subfractions_Apo.A1_HDL.4 & 54.24 [48.105 57.045] & $64.89[60.08576 .18]$ & -0.35 & $2.67 \times 10^{-3}$ & $6.10 \times 10^{-2}$ \\
\hline LDL_Subfractions_Triglycerides_LDL.1 & 8.15 [7.56 9.31] & $5.15[3.77 .2]$ & 0.57 & $4.45 \times 10^{-3}$ & $8.45 \times 10^{-2}$ \\
\hline HDL_Subfractions_Phospholipid_HDL.4 & 18.97 [18.37 19.995] & $22.74[20.58$ 27.65] & -0.33 & $5.22 \times 10^{-3}$ & $8.50 \times 10^{-2}$ \\
\hline MainFractions_Triglycerides_LDL & 25.6 [23.305 28.935] & 20.07 [16.775 23.18] & 0.38 & $8.27 \times 10^{-3}$ & $1.18 \times 10^{-1}$ \\
\hline MainFractions_Phospholipids_IDL & $2.88[2.15 .535]$ & 7.1 [4.77 11.555] & -1.13 & $9.37 \times 10^{-3}$ & $1.19 \times 10^{-1}$ \\
\hline HDL_Subfractions_Cholesterol_HDL.4 & 13.87 [11.825 14.56] & 18.07 [14.09 22.105] & -0.44 & $1.06 \times 10^{-2}$ & $1.21 \times 10^{-1}$ \\
\hline VLDL_Subfractions_Cholesterol_VLDL.1 & 2.71 [1.69 4.625] & 7.76 [4.5 17.16] & -1.52 & $1.43 \times 10^{-2}$ & $1.48 \times 10^{-1}$ \\
\hline Main_Parameters_Cholesterol & 151.07 [139.25 184.675] & 209.08 [183.615 223.545] & -0.32 & $1.89 \times 10^{-2}$ & $1.78 \times 10^{-1}$ \\
\hline VLDL_Subfractions_FreeCholesterol_VLDL.1 & $0.5[0.3551 .135]$ & $2.84[1.3456 .68]$ & -2.05 & $2.02 \times 10^{-2}$ & $1.78 \times 10^{-1}$ \\
\hline LDL.5_Particle_Number & $122.22[44.215173 .705]$ & 235.58 [150.9 303.1] & -0.82 & $2.75 \times 10^{-2}$ & $2.08 \times 10^{-1}$ \\
\hline LDL_Subfractions_Apo.B_LDL.5 & $6.72[2.439 .555]$ & $12.96[8.316 .67]$ & -0.82 & $2.75 \times 10^{-2}$ & $2.08 \times 10^{-1}$ \\
\hline VLDL_Subfractions_Phospholipids_VLDL.1 & 2.89 [2.05 3.805] & 7.51 [3.32 14.185] & -1.4 & $3.09 \times 10^{-2}$ & $2.08 \times 10^{-1}$ \\
\hline VLDL_Subfractions_Cholesterol_VLDL.2 & 2.38 [1.82 2.735] & $3.91[2.9355 .69]$ & -0.73 & $3.32 \times 10^{-2}$ & $2.08 \times 10^{-1}$ \\
\hline LDL_S̄ubfractions_C̄holesterol_L̄DL.5 & $7.27[1.46511 .7]$ & 18.74 [10.945 22.83] & -1.01 & $3.32 \times 10^{-2}$ & $2.08 \times 10^{-1}$ \\
\hline LDL_Subfractions_Phospholipids_LDL.5 & 4.84 [1.515 6.935] & 9.85 [6.405 11.875] & -0.87 & $3.47 \times 10^{-2}$ & $2.08 \times 10^{-1}$ \\
\hline VLDL_Subfractions_Triglycerides_VLDL.1 & $11.87[9.4226 .39]$ & 39.89 [19.25 92.63] & -1.53 & $4.35 \times 10^{-2}$ & $2.36 \times 10^{-1}$ \\
\hline HDL_Subfractions_Triglycerides_HDL.1 & $4.34[3.7855 .4]$ & 3.33 [2.37 3.97] & 0.4 & $4.35 \times 10^{-2}$ & $2.36 \times 10^{-1}$ \\
\hline MainFractions_Apo.A1_HDL & 126.51 [118.6 131.56] & 138.97 [133.715 147.975] & -0.14 & $4.85 \times 10^{-2}$ & $2.51 \times 10^{-1}$ \\
\hline Main_Parameters_Apo.A1 & 128.49 [120.11 132.985] & 137.99 [131.55 147.33] & -0.13 & $5.98 \times 10^{-2}$ & $2.97 \times 10^{-1}$ \\
\hline LDL_Subfractions_FreeCholesterol_LDL.5 & $2.63[1.053 .635]$ & $4.45[2.985 .795]$ & -0.66 & $7.36 \times 10^{-2}$ & $3.50 \times 10^{-1}$ \\
\hline HDL_Subfractions_Triglycerides_HDL.2 & $2.14\left[\begin{array}{ll}1.96 & 2.345\end{array}\right]$ & $1.7\left[\begin{array}{lll}1.365 & 2.22\end{array}\right]$ & 0.32 & $8.07 \times 10^{-2}$ & $3.68 \times 10^{-1}$ \\
\hline LDL_Subfractions_Triglycerides_LDL.3 & $2.9[2.513 .37]$ & 2.4 [1.705 2.765] & 0.36 & $8.45 \times 10^{-2}$ & $3.68 \times 10^{-1}$ \\
\hline LDL_Subfractions_Cholesterol_LDL.4 & 1.51 [0 4.95] & 8.94 [2.735 13.525] & -1.03 & $8.71 \times 10^{-2}$ & $3.68 \times 10^{-1}$ \\
\hline MainFractions_Triglycerides_IDL & $4.43[2.8957 .345]$ & $9.89[5.21525 .96]$ & -1.39 & $9.25 \times 10^{-2}$ & $3.70 \times 10^{-1}$ \\
\hline Main_Parameters_LDL.Chol & 70.08 [51.52 95.205] & $104.2[82.98$ 116.18] & -0.38 & $9.74 \times 10^{-2}$ & $3.70 \times 10^{-1}$ \\
\hline MainFractions_Cholesterol_LDL & 70.08 [51.52 95.205] & 104.2 [82.98 116.18] & -0.38 & $9.74 \times 10^{-2}$ & $3.70 \times 10^{-1}$ \\
\hline LDL_Subfractions_Triglycerides_LDL.4 & $2.58[2.162 .75]$ & 1.55 [1.11 2.14] & 0.56 & $1.06 \times 10^{-1}$ & $3.88 \times 10^{-1}$ \\
\hline MainFractions_Phospholipids_VLDL & $14.07[11.88523 .41]$ & $23.72[16.43539 .41]$ & -0.56 & $1.17 \times 10^{-1}$ & $3.95 \times 10^{-1}$ \\
\hline LDL.4_Particle_Number & 34.66 [0 72.185] & 119.2 [41.125 153.81] & -0.85 & $1.24 \times 10^{-1}$ & $3.95 \times 10^{-1}$ \\
\hline LDL_Subfractions_Phospholipids_LDL.4 & $2.14[03.865]$ & $5.41[1.777 .75]$ & -0.82 & $1.24 \times 10^{-1}$ & $3.95 \times 10^{-1}$ \\
\hline LDL_Subfractions_Apo.B_LDL.4 & $1.91[03.97]$ & $6.56[2.2658 .46]$ & -0.85 & $1.24 \times 10^{-1}$ & $3.95 \times 10^{-1}$ \\
\hline MainFractions_FreeCholesterol_VLDL & $6.27[5.15510 .92]$ & $11.05\left[\begin{array}{l}7.74 \\
17.9]\end{array}\right.$ & -0.58 & $1.25 \times 10^{-1}$ & $3.95 \times 10^{-1}$ \\
\hline LDL.Chol_HDL.Chol & $1.48[0.9851 .8]$ & 1.82 [1.61 2.12] & -0.23 & $1.47 \times 10^{-1}$ & $3.95 \times 10^{-1}$ \\
\hline MainFractions_Cholesterol_VLDL & 13.65 [11.82 24.19] & 26.46 [17.545 40.495] & -0.57 & $1.51 \times 10^{-1}$ & $3.95 \times 10^{-1}$ \\
\hline LDL_Subfractions_Phospholipids_LDL.6 & 12.38 [9.875 17.385] & 16.01 [13.97 19.735] & -0.31 & $1.51 \times 10^{-1}$ & $3.95 \times 10^{-1}$ \\
\hline HDL_Subfractions_Phospholipid_HDL.1 & $25.88[23.35528 .415]$ & 20.69 [14.64 27.49] & 0.29 & $1.51 \times 10^{-1}$ & $3.95 \times 10^{-1}$ \\
\hline MainFractions_FreeCholesterol_LDL & 22.25 [19.255 27.525] & $28.41[24.24532 .88]$ & -0.21 & $1.60 \times 10^{-1}$ & $3.95 \times 10^{-1}$ \\
\hline Main_Parameters_Apo.B100 & 69.62 [62.005 86.355] & 91.46 [76.055 99.625] & -0.21 & $1.63 \times 10^{-1}$ & $3.95 \times 10^{-1}$ \\
\hline Total_Particle_Number & 1265.89 [1127.435 1570.115] & 1663.05 [1382.915 1811.48] & -0.21 & $1.63 \times 10^{-1}$ & $3.95 \times 10^{-1}$ \\
\hline MainFractions_Triglycerides_VLDL & $60.34[40.1472 .935]$ & $74.42[49.29161 .715]$ & -0.77 & $1.63 \times 10^{-1}$ & $3.95 \times 10^{-1}$ \\
\hline LDL_Subfractions_Cholesterol_LDL.6 & 21.31 [16.14 31.8] & 29.34 [24.955 37.035] & -0.38 & $1.63 \times 10^{-1}$ & $3.95 \times 10^{-1}$ \\
\hline LDL_Subfractions_Apo.B_LDL.1 & 13.34 [12.155 13.715] & 11.37 [8.335 13.33] & 0.18 & $1.73 \times 10^{-1}$ & $3.95 \times 10^{-1}$ \\
\hline LDL_Particle_Number & 1006.8 [780.95 1357.535] & $\begin{array}{c}1329.88[1132.015 \\
1424.785]\end{array}$ & -0.23 & $1.77 \times 10^{-1}$ & $3.95 \times 10^{-1}$ \\
\hline LDL.1_Particle_Number & 242.55 [220.995 249.37] & 206.72 [151.57 242.355] & 0.18 & $1.77 \times 10^{-1}$ & $3.95 \times 10^{-1}$ \\
\hline MainFractions_Phospholipids_LDL & 46.19 [37.155 58.085] & 59.18 [47.095 66.04] & -0.2 & $1.77 \times 10^{-1}$ & $3.95 \times 10^{-1}$ \\
\hline MainFractions_Apo.B_LDL & $55.37[42.9574 .66]$ & $73.14[62.25578 .36]$ & -0.23 & $1.77 \times 10^{-1}$ & $3.95 \times 10^{-1}$ \\
\hline HDL_Subfractions_Cholesterol_HDL.1 & 22.1 [19.685 24.495] & $18.78[13.85523 .415]$ & 0.24 & $1.77 \times 10^{-1}$ & $3.95 \times 10^{-1}$ \\
\hline VLDL_Subfractions_FreeCholesterol_VLDL.2 & 1.17 [1.04 1.43] & 1.91 [1.16 2.945] & -0.58 & $1.87 \times 10^{-1}$ & $4.09 \times 10^{-1}$ \\
\hline MainFractions_FreeCholesterol_IDL & 2.34 [1.975 4.385] & $4.03[2.785 .695]$ & -0.46 & $2.01 \times 10^{-1}$ & $4.28 \times 10^{-1}$ \\
\hline LDL.6_Particle_Number & $325.77[240.52484 .46]$ & $450.55[346.56564 .44]$ & -0.39 & $2.06 \times 10^{-1}$ & $4.28 \times 10^{-1}$ \\
\hline LDL_Subfractions_Apo.B_LDL.6 & 17.92 [13.23 26.645] & 24.78 [19.06 31.045] & -0.39 & $2.06 \times 10^{-1}$ & $4.28 \times 10^{-1}$ \\
\hline VLDL_Subfractions_Phospholipids_VLDL.2 & $2.64[2.473 .195]$ & $3.8[2.4655 .65]$ & -0.47 & $2.17 \times 10^{-1}$ & $4.41 \times 10^{-1}$ \\
\hline Main_Parameters_Triglycerides & 96.35 [80.635 122.505] & 114.96 [91.02 219.825] & -0.57 & $2.39 \times 10^{-1}$ & $4.78 \times 10^{-1}$ \\
\hline MainFractions_Triglycerides_HDL & $10.91[9.75511 .665]$ & $8.54[7.1312 .12]$ & 0.14 & $2.57 \times 10^{-1}$ & $4.92 \times 10^{-1}$ \\
\hline LDL_Subfractions_FreeCholesterol_LDL.4 & 1.95 [0.545 2.795] & 2.95 [1.57 4.345] & -0.5 & $2.59 \times 10^{-1}$ & $4.92 \times 10^{-1}$ \\
\hline HDL_Subfractions_Apo.A2_HDL.3 & $5.51[5.1655 .945]$ & $6.01[5.5356 .885]$ & -0.19 & $2.59 \times 10^{-1}$ & $4.92 \times 10^{-1}$ \\
\hline MainFractions_Cholesterol_IDL & $9.21[7.73515 .675]$ & $14.04[9.7118 .84]$ & -0.35 & $2.75 \times 10^{-1}$ & $5.14 \times 10^{-1}$ \\
\hline LDL_Subfractions_FreeCholesterol_LDL.1 & $6.71[5.867 .34]$ & $5.47[4.4957 .21]$ & 0.11 & $2.87 \times 10^{-1}$ & $5.28 \times 10^{-1}$ \\
\hline VLDL_Subfractions_FreeCholesterol_VLDL.5 & $0.39[0.351 .16]$ & $0.93[0.5751 .565]$ & -0.59 & $2.94 \times 10^{-1}$ & $5.32 \times 10^{-1}$ \\
\hline LDL_Subfractions_Phospholipids_LDL.1 & $13.28[11.5713 .34]$ & $11.24[8.92513 .96]$ & 0.1 & $3.07 \times 10^{-1}$ & $5.46 \times 10^{-1}$ \\
\hline Main_Parameters_HDL.Chol & 52.7 [48.33 54.175] & 55.73 [51.2 62.19] & -0.1 & $3.35 \times 10^{-1}$ & $5.79 \times 10^{-1}$ \\
\hline
\end{tabular}


MainFractions_Cholesterol_HDL

LDL_Subfractions_FreeCholesterol_LDL.6

LDL_Subfractions_FreeCholesterol_LDL.2

HDL_Subfractions_Triglycerides_HDL.3

HDL_Subfractions_FreeCholesterol_HDL.3

VLDL_Subfractions_Triglycerides_VLDL.2

VLDL_Subfractions_Triglycerides_VLDL.4

HDL_Subfractions_Triglycerides_HDL.4

VLDL_Subfractions_FreeCholesterol_VLDL.3

Apo.B100_Apo.A1

VLDL_Subfractions_Cholesterol_VLDL.3

VLDL_Subfractions_Triglycerides_VLDL.5

HDL_Subfractions_FreeCholesterol_HDL.2

HDL_Subfractions_Apo.A2_HDL.1

HDL_Subfractions_Cholesterol_HDL.3

LDL_Subfractions_Triglycerides_LDL.6

HDL_Subfractions_FreeCholesterol_HDL.4

VLDL_Subfractions_Phospholipids_VLDL.3

LDL.2 Particle Number

LDL_Subfractions_Apo.B_LDL.2

VLDL_Subfractions_FreeCholesterol_VLDL.4

LDL_Subfractions_Triglycerides_LDL.5

HDL_Subfractions_Apo.A1_HDL.1

VLDL_Subfractions_Cholesterol_VLDL.4

VLDL_Subfractions_Cholesterol_VLDL.5

VLDL_Particle_Number

IDL_Particle_Number

MainFractions_Apo.B_VLDL

MainFractions_Apo.B_IDL

LDL_Subfractions_Cholesterol_LDL.3

MainFractions_FreeCholesterol_HDL

HDL_Subfractions_FreeCholesterol_HDL.1

LDL_Subfractions_Phospholipids_LDL.2

LDL_Subfractions_Apo.B_LDL.3

LDL.3_Particle_Number

LDL_Subfractions_FreeCholesterol_LDL.3

LDL_Subfractions_Cholesterol_LDL.2

HDL Subfractions Apo.A1 HDL.3

HDL_Subfractions_Phospholipid_HDL.2

VLDL_Subfractions_Phospholipids_VLDL.5

HDL_Subfractions_Apo.A1_HDL.2

VLDL_Subfractions_Phospholipids_VLDL.4

LDL_Subfractions_Phospholipids_LDL.3

HDL_Subfractions_Cholesterol_HDL.2

VLDL_Subfractions_Triglycerides_VLDL.3

MainFractions Phospholipids HDL

LDL_Subfractions_Cholesterol_LDL.1

HDL Subfractions Phospholipid HDL.3

HDL_Subfractions_Apo.A2_HDL.2

$$
\begin{aligned}
& 52.7 \text { [48.33 54.175] } \\
& 5.21 \text { [3.14 7.33] } \\
& 5.75 \text { [5.19 6.645] } \\
& 2.21 \text { [2.09 2.45] } \\
& 1.4 \text { [1.325 1.605] } \\
& 9.48 \text { [9.145 12.295] } \\
& 11.24 \text { [8.685 16.995] } \\
& 2.8 \text { [2.31 3.11] } \\
& 1.11 \text { [1.075 2.275] } \\
& 0.57 \text { [0.475 0.68] } \\
& 2.67 \text { [2.43 5.15] } \\
& 3.31 \text { [2.555 3.66] } \\
& 1.75 \text { [1.495 1.82] } \\
& 2.93 \text { [2.72 3.645] } \\
& 8.81 \text { [8.215 9.26] } \\
& 5.11 \text { [4.85 7.015] } \\
& 2.4 \text { [2.05 2.655] } \\
& 3.31 \text { [2.965 5.41] } \\
& 166.03 \text { [151.33 208.055] } \\
& 9.13 \text { [8.32 11.44] } \\
& 1.52 \text { [1.075 3.665] } \\
& 2.56 \text { [2.13.05] } \\
& 27.97 \text { [26.28 30.92] } \\
& 4.74 \text { [3.605 8.725] } \\
& 1.21[0.591 .64] \\
& 146.61 \text { [124.635 216.05] } \\
& 85.1 \text { [82.795 107.41] } \\
& 8.06 \text { [6.855 11.885] } \\
& 4.68 \text { [4.555 5.905] } \\
& 9.6 \text { [6.69 12.495] } \\
& 10.57 \text { [10.04 12.075] } \\
& 3.98 \text { [3.115 4.53] } \\
& 8.86 \text { [8.125 10.94] } \\
& 6.96 \text { [5.93 8.795] } \\
& 126.58 \text { [107.8 159.865] } \\
& 3.79 \text { [3.595 4.735] } \\
& 14.59 \text { [13.305 19.375] } \\
& 24.55 \text { [22.595 25.55] } \\
& 13.27 \text { [12.895 14.535] } \\
& 1.36 \text { [1 2.415] } \\
& 19.84 \text { [15.235 20.365] } \\
& 4.86 \text { [3.69 7.7] } \\
& 6.36[5.47 .79] \\
& 8.36 \text { [7.58 8.71] } \\
& 9.86 \text { [9.56 16.12] } \\
& 73.02 \text { [67.19 75.64] } \\
& 20.18 \text { [17.485 21.32] } \\
& 14.39 \text { [13.985 15.93] } \\
& 3.83 \text { [3.415 4.015] }
\end{aligned}
$$

\begin{tabular}{|c|c|c|c|}
\hline 55.73 [51.2 62.19] & -0.1 & $3.35 \times 10^{-1}$ & $5.79 \times 10^{-1}$ \\
\hline $6.48[5.0457 .83]$ & -0.27 & $3.49 \times 10^{-1}$ & $5.90 \times 10^{-1}$ \\
\hline $5.35[4.1856 .4]$ & 0.18 & $3.57 \times 10^{-1}$ & $5.90 \times 10^{-1}$ \\
\hline 1.71 [ 1.585 2.645] & 0.17 & $3.57 \times 10^{-1}$ & $5.90 \times 10^{-1}$ \\
\hline 1.67 [1.375 1.95] & -0.1 & $3.71 \times 10^{-1}$ & $6.04 \times 10^{-1}$ \\
\hline 12.3 [7.77 20.28] & -0.35 & $4.03 \times 10^{-1}$ & $6.26 \times 10^{-1}$ \\
\hline 10.15 [7.525 13.39] & 0.26 & $4.03 \times 10^{-1}$ & $6.26 \times 10^{-1}$ \\
\hline $2.86[2.5153 .98]$ & -0.23 & $4.03 \times 10^{-1}$ & $6.26 \times 10^{-1}$ \\
\hline 2.12 [1.16 3.395] & -0.42 & $4.06 \times 10^{-1}$ & $6.26 \times 10^{-1}$ \\
\hline $0.66\left[\begin{array}{lll}0.56 & 0.745\end{array}\right]$ & -0.06 & $4.18 \times 10^{-1}$ & $6.35 \times 10^{-1}$ \\
\hline $4.67[2.66$ 6.615] & -0.32 & $4.56 \times 10^{-1}$ & $6.84 \times 10^{-1}$ \\
\hline 3.01 [2.105 3.655] & 0.19 & $4.69 \times 10^{-1}$ & $6.94 \times 10^{-1}$ \\
\hline 1.57 [1.03 1.81] & 0.1 & $5.23 \times 10^{-1}$ & $7.55 \times 10^{-1}$ \\
\hline 2.86 [2.115 3.64] & 0.12 & $5.23 \times 10^{-1}$ & $7.55 \times 10^{-1}$ \\
\hline 9 [8.4 10.165] & -0.05 & $5.31 \times 10^{-1}$ & $7.55 \times 10^{-1}$ \\
\hline 5.12 [4.355 6.655] & 0.11 & $5.37 \times 10^{-1}$ & $7.55 \times 10^{-1}$ \\
\hline $2.42[1.693 .47]$ & -0.22 & $5.59 \times 10^{-1}$ & $7.76 \times 10^{-1}$ \\
\hline $4.97[3.116 .755]$ & -0.22 & $5.65 \times 10^{-1}$ & $7.76 \times 10^{-1}$ \\
\hline 4.37 [139.665 205.405] & 0.11 & $5.88 \times 10^{-1}$ & $7.84 \times 10^{-1}$ \\
\hline 9.04 [7.68 11.295] & 0.11 & $5.88 \times 10^{-1}$ & $7.84 \times 10^{-1}$ \\
\hline 2.14 [1.405 3.315] & -0.14 & $6.09 \times 10^{-1}$ & $7.84 \times 10^{-1}$ \\
\hline 2.94 [2.205 3.695] & -0.07 & $6.09 \times 10^{-1}$ & $7.84 \times 10^{-1}$ \\
\hline 26.72 [18.345 36.18] & 0.15 & $6.17 \times 10^{-1}$ & $7.84 \times 10^{-1}$ \\
\hline $5.18[4.1657 .74]$ & 0 & $6.39 \times 10^{-1}$ & $7.84 \times 10^{-1}$ \\
\hline $1.51[0.841 .88]$ & -0.06 & $6.39 \times 10^{-1}$ & $7.84 \times 10^{-1}$ \\
\hline 4.76 [125.635 260.425] & -0.19 & $6.47 \times 10^{-1}$ & $7.84 \times 10^{-1}$ \\
\hline 3.81 [66.715 125.515] & -0.12 & $6.47 \times 10^{-1}$ & $7.84 \times 10^{-1}$ \\
\hline 9.61 [6.91 14.32] & -0.19 & $6.47 \times 10^{-1}$ & $7.84 \times 10^{-1}$ \\
\hline $5.16[3.676 .9]$ & -0.12 & $6.47 \times 10^{-1}$ & $7.84 \times 10^{-1}$ \\
\hline 13.12 [7.465 15.125] & -0.13 & $6.70 \times 10^{-1}$ & $8.04 \times 10^{-1}$ \\
\hline 9.66 [9.24 12.95] & 0.06 & $7.01 \times 10^{-1}$ & $8.24 \times 10^{-1}$ \\
\hline 3.75 [2.475 4.825] & 0.02 & $7.01 \times 10^{-1}$ & $8.24 \times 10^{-1}$ \\
\hline 8.8 [7.225 11.315] & 0.09 & $7.39 \times 10^{-1}$ & $8.60 \times 10^{-1}$ \\
\hline 7.25 [5.035 9.525] & 0.11 & $7.66 \times 10^{-1}$ & $8.79 \times 10^{-1}$ \\
\hline 31.79 [91.545 173.235] & 0.11 & $7.71 \times 10^{-1}$ & $8.79 \times 10^{-1}$ \\
\hline $4.21[2.535 .035]$ & 0.15 & $7.98 \times 10^{-1}$ & $8.97 \times 10^{-1}$ \\
\hline 16.89 [13.345 20.83] & -0.01 & $8.03 \times 10^{-1}$ & $8.97 \times 10^{-1}$ \\
\hline 23.88 [22.23 27.09] & -0.05 & $8.15 \times 10^{-1}$ & $9.02 \times 10^{-1}$ \\
\hline $14.26[10.64$ 15.715] & 0.07 & $8.35 \times 10^{-1}$ & $9.15 \times 10^{-1}$ \\
\hline 1.97 [1.055 2.29] & -0.06 & $8.48 \times 10^{-1}$ & $9.21 \times 10^{-1}$ \\
\hline 17.65 [15.25 20.59] & 0.02 & $8.98 \times 10^{-1}$ & $9.66 \times 10^{-1}$ \\
\hline 4.87 [3.93 6.625] & 0.1 & $9.15 \times 10^{-1}$ & $9.75 \times 10^{-1}$ \\
\hline $7.63[4.788 .565]$ & 0.05 & $9.32 \times 10^{-1}$ & $9.76 \times 10^{-1}$ \\
\hline 8.56 [6.67 10.025] & -0.01 & $9.34 \times 10^{-1}$ & $9.76 \times 10^{-1}$ \\
\hline 11.94 [9.045 19.305] & -0.03 & $9.66 \times 10^{-1}$ & $9.84 \times 10^{-1}$ \\
\hline 72.67 [65.82 80.31] & -0.01 & $9.67 \times 10^{-1}$ & $9.84 \times 10^{-1}$ \\
\hline 18.55 [14.815 24.82] & -0.07 & $9.67 \times 10^{-1}$ & $9.84 \times 10^{-1}$ \\
\hline 14.33 [13.63 16.59] & 0 & $9.83 \times 10^{-1}$ & $9.92 \times 10^{-1}$ \\
\hline 3.61 [3.125 4.435] & -0.06 & 1.00 & 1.00 \\
\hline
\end{tabular}

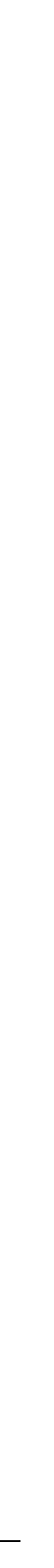


439 Table S3. Statistical comparison between the metabolic profiles of Metabotype II versus Metabotype III.

\begin{tabular}{|c|c|c|c|c|c|}
\hline Feature & Metabotype II, median [IQR] & Metabotype III, median [IQR] & log change & p-value & FDR \\
\hline Pyroglutamate & $1.28[1.2261 .343]$ & $0.974[0.9431 .011]$ & 0.45 & $1.02 \times 10^{-6}$ & $1.05 \times 10^{-5}$ \\
\hline 2-Hydroxyvalerate & $1.263[1.211 .325]$ & 0.961 [0.93 0.997] & 0.45 & $1.02 \times 10^{-6}$ & $1.05 \times 10^{-5}$ \\
\hline Unsaturated lipid $(-\mathrm{CH}=\mathrm{CH}-)$ & $1.388\left[\begin{array}{lll}1.331 & 1.469\end{array}\right]$ & $0.917\left[\begin{array}{lll}0.838 & 0.998\end{array}\right]$ & 0.64 & $1.02 \times 10^{-6}$ & $1.05 \times 10^{-5}$ \\
\hline Lipid (-(-CH2-)n-) & 1.569 [1.51 1.742] & 0.892 [0.776 1.003] & 0.91 & $1.02 \times 10^{-6}$ & $1.05 \times 10^{-5}$ \\
\hline Lipid (-CH3-) & $1.226\left[\begin{array}{lll}1.183 & 1.272\end{array}\right]$ & $0.948[0.9381 .034]$ & 0.33 & $2.04 \times 10^{-6}$ & $1.67 \times 10^{-5}$ \\
\hline Lipid (alpha-CH2) & $2.144[1.9572 .631]$ & $0.671[0.4940 .863]$ & 1.76 & $7.14 \times 10^{-6}$ & $4.18 \times 10^{-5}$ \\
\hline Glycorol phospholipid & $2.084[1.919$ 2.643] & $0.552\left[\begin{array}{lll}0.401 & 0.809\end{array}\right]$ & 1.73 & $7.14 \times 10^{-6}$ & $4.18 \times 10^{-5}$ \\
\hline Threonine & $1.596[1.341$ 1.786] & $0.783[0.7410 .96]$ & 0.96 & $4.59 \times 10^{-5}$ & $2.35 \times 10^{-4}$ \\
\hline GlycB & $0.72[0.6610 .783]$ & 0.978 [0.903 1.167] & -0.5 & $1.42 \times 10^{-4}$ & $6.46 \times 10^{-4}$ \\
\hline Lipid (beta-CH2) & $1.288[1.1931 .36]$ & 1.032 [0.9 1.121] & 0.36 & $1.98 \times 10^{-4}$ & $8.11 \times 10^{-4}$ \\
\hline 3-Hydroxybutyrate & $1.28\left[\begin{array}{ll}1.183 & 1.351\end{array}\right]$ & 0.979 [0.949 1.017] & 0.4 & $3.73 \times 10^{-4}$ & $1.39 \times 10^{-3}$ \\
\hline Lipid (=CH-CH2-CH=) & $1.111[1.081 .156]$ & 0.957 [0.818 1.046] & 0.28 & $1.49 \times 10^{-3}$ & $5.10 \times 10^{-3}$ \\
\hline Cholesterol backbone & 1.324 [1.228 1.43] & 1.021 [0.851 1.149] & 0.37 & $1.91 \times 10^{-3}$ & $6.03 \times 10^{-3}$ \\
\hline Methanol & 0.978 [0.826 1.148] & 1.41 [1.091 1.572] & -0.46 & $2.20 \times 10^{-2}$ & $6.45 \times 10^{-2}$ \\
\hline Pyruvate & 1.362 [0.789 1.844] & 0.918 [0.581 1.09] & 0.75 & $4.17 \times 10^{-2}$ & $1.14 \times 10^{-1}$ \\
\hline Phenylalanine & $1.003[0.9421 .072]$ & $0.834[0.6691 .01]$ & 0.28 & $4.84 \times 10^{-2}$ & $1.24 \times 10^{-1}$ \\
\hline Isoleucine & 1.146 [1.062 1.254] & 0.911 [0.777 1.182] & 0.28 & $8.41 \times 10^{-2}$ & $2.03 \times 10^{-1}$ \\
\hline GlycA & 1.019 [0.96 1.073] & 0.913 [0.827 0.979] & 0.13 & $9.56 \times 10^{-2}$ & $2.18 \times 10^{-1}$ \\
\hline Creatine & 1.401 [0.958 1.613] & 0.931 [0.45 1.289] & 0.33 & $1.08 \times 10^{-1}$ & $2.34 \times 10^{-1}$ \\
\hline Mannose & 1.11 [0.949 1.27] & 0.977 [0.714 1.076] & 0.25 & $1.22 \times 10^{-1}$ & $2.51 \times 10^{-1}$ \\
\hline Valine & $1.01[0.9361 .284]$ & $0.956[0.8061 .069]$ & 0.19 & $1.38 \times 10^{-1}$ & $2.68 \times 10^{-1}$ \\
\hline 3-Hydroxyisovalerate & 0.568 [0.479 0.635] & 1.799 [0.498 4.786] & -1.53 & $1.72 \times 10^{-1}$ & $3.21 \times 10^{-1}$ \\
\hline Glucose & 1.011 [0.925 1.471] & 0.911 [0.889 1.124] & 0.28 & $1.92 \times 10^{-1}$ & $3.41 \times 10^{-1}$ \\
\hline Glutamine & $1.157\left[\begin{array}{lll}1.108 & 1.204]\end{array}\right]$ & 1.07 [0.999 1.213] & 0.07 & $2.35 \times 10^{-1}$ & $4.01 \times 10^{-1}$ \\
\hline Acetate & 0.956 [0.895 1.125] & 1.149 [0.959 1.291] & -0.15 & $3.41 \times 10^{-1}$ & $5.59 \times 10^{-1}$ \\
\hline Formate & 0.979 [0.758 1.077] & $1.051[0.7161 .253]$ & -0.18 & $3.71 \times 10^{-1}$ & $5.85 \times 10^{-1}$ \\
\hline Glutamate & $0.802[0.461 .302]$ & 1.175 [0.814 1.223] & -0.23 & $4.03 \times 10^{-1}$ & $6.12 \times 10^{-1}$ \\
\hline Glycine & 1.083 [0.933 1.158] & 1.151 [0.99 1.195] & -0.1 & $4.37 \times 10^{-1}$ & $6.39 \times 10^{-1}$ \\
\hline Histidine & $1.101[1.0291 .16]$ & 1.053 [0.967 1.151] & 0.04 & $4.72 \times 10^{-1}$ & $6.67 \times 10^{-1}$ \\
\hline Creatinine & $1.023[0.9061 .071]$ & $0.922[0.7541 .126]$ & 0.09 & $5.08 \times 10^{-1}$ & $6.72 \times 10^{-1}$ \\
\hline Leucine & 1.069 [0.941 1.199] & 0.94 [0.842 1.145] & 0.1 & $5.08 \times 10^{-1}$ & $6.72 \times 10^{-1}$ \\
\hline Tyrosine & 1.044 [0.928 1.109] & $0.918[0.7841 .1]$ & 0.11 & $5.46 \times 10^{-1}$ & $6.99 \times 10^{-1}$ \\
\hline Alanine & 1.019 [0.856 1.274] & $0.95[0.8791 .126]$ & 0.16 & $5.85 \times 10^{-1}$ & $7.27 \times 10^{-1}$ \\
\hline 2-Hydroxybutyrate & $1.024[0.7461 .18]$ & $0.865[0.8061 .063]$ & 0.04 & $7.96 \times 10^{-1}$ & $9.31 \times 10^{-1}$ \\
\hline Protein & 1.013 [0.962 1.072] & 1.028 [0.993 1.064] & 0 & $7.96 \times 10^{-1}$ & $9.31 \times 10^{-1}$ \\
\hline Desaminotyrosine & $1\left[\begin{array}{ll}1 & 1\end{array}\right]$ & $1\left[\begin{array}{ll}1 & 1\end{array}\right]$ & -1.32 & $8.37 \times 10^{-1}$ & $9.31 \times 10^{-1}$ \\
\hline Citrate.left & 1.003 [0.778 1.279] & 1.035 [0.819 1.249] & -0.01 & $8.41 \times 10^{-1}$ & $9.31 \times 10^{-1}$ \\
\hline Lactate & 0.79 [0.689 1.483] & 0.82 [0.709 1.035] & 0.21 & $8.86 \times 10^{-1}$ & $9.31 \times 10^{-1}$ \\
\hline Acetoacetate & $0.943[0.7241 .231]$ & 0.904 [0.659 1.244] & -0.18 & $8.86 \times 10^{-1}$ & $9.31 \times 10^{-1}$ \\
\hline Phospholipid & 1.006 [0.925 1.143] & 1.035 [0.934 1.099] & -0.02 & $9.31 \times 10^{-1}$ & $9.55 \times 10^{-1}$ \\
\hline Isobutyrate & 0.972 [0.895 1.035] & 0.918 [0.817 1.3] & -0.15 & $9.77 \times 10^{-1}$ & $9.77 \times 10^{-1}$ \\
\hline
\end{tabular}




\begin{tabular}{|c|c|c|c|c|c|}
\hline Feature & Metabotype II, median [IQR] & Metabotype III, median [IQR] & log change & p-value & FDR \\
\hline Main_Parameters_Triglycerides & $240.36[214.002299 .433]$ & $91.02[71.323111 .78]$ & 1.52 & $2.04 \times 10^{-6}$ & $8.14 \times 10^{-5}$ \\
\hline MainFractions_Triglycerides_VLDL & 170.69 [159.183 210.132] & $49.29[38.68869 .142]$ & 1.79 & $4.08 \times 10^{-6}$ & $8.14 \times 10^{-5}$ \\
\hline MainFractions_Triglycerides_IDL & 26.57 [25.95 36.155] & $5.215[2.488 .28]$ & 2.47 & $4.08 \times 10^{-6}$ & $8.14 \times 10^{-5}$ \\
\hline VLDL_Subfractions_Triglycerides_VLDL.1 & 107.94 [80.3 134.335] & 19.25 [11.785 27.652] & 2.45 & $4.08 \times 10^{-6}$ & $8.14 \times 10^{-5}$ \\
\hline VLDL_Subfractions_Phospholipids_VLDL.1 & 15.77 [13.717 19.802] & $3.32[2.454 .475]$ & -2.2 & $4.08 \times 10^{-6}$ & $8.14 \times 10^{-5}$ \\
\hline VLDL_Particle_Number & 278.955 [243.442 330.25] & 125.635 [95.94 150.235] & 1.26 & $7.14 \times 10^{-6}$ & $8.14 \times 10^{-5}$ \\
\hline MainFractions_Cholesterol_VLDL & $43.075[37.28554 .905]$ & $17.545[11.70721 .458]$ & 1.42 & $7.14 \times 10^{-6}$ & $8.14 \times 10^{-5}$ \\
\hline MainFractions_Cholesterol_IDL & $18.84[15.55328 .68]$ & 9.94 [6.973 12.57] & 1.22 & $7.14 \times 10^{-6}$ & $8.14 \times 10^{-5}$ \\
\hline MainFractions_Apo.B_VLDL & 15.34 [13.39 18.163] & $6.91[5.2758 .262]$ & 1.26 & $7.14 \times 10^{-6}$ & $8.14 \times 10^{-5}$ \\
\hline VLDL_Subfractions_Cholesterol_VLDL.1 & $20.4[16.01725 .052]$ & 4.5 [2.473 6.945] & 2.2 & $7.14 \times 10^{-6}$ & $8.14 \times 10^{-5}$ \\
\hline Main_Parameters_Apo.B100 & 105.6 [96.73 118.345] & 80.055 [67.373 86.227] & 0.45 & $3.06 \times 10^{-5}$ & $2.49 \times 10^{-4}$ \\
\hline Total_Particle_Number & 1920.06 [1758.775 2151.845] & 1455.6 [1224.988 1567.833] & 0.45 & $3.06 \times 10^{-5}$ & $2.49 \times 10^{-4}$ \\
\hline MainFractions_Phospholipids_VLDL & 43.66 [36.663 48.425] & 16.435 [12.775 21.26] & 1.35 & $3.06 \times 10^{-5}$ & $2.49 \times 10^{-4}$ \\
\hline VLDL_Subfractions_Triglycerides_VLDL.2 & 23.955 [18.545 28.067] & 7.77 [6.612 12.015] & 1.31 & $3.06 \times 10^{-5}$ & $2.49 \times 10^{-4}$ \\
\hline VLDL_Subfractions_FreeCholesterol_VLDL.3 & $3.745[3.1654 .645]$ & $1.16[0.6621 .658]$ & 1.65 & $4.59 \times 10^{-5}$ & $3.27 \times 10^{-4}$ \\
\hline VLDL_Subfractions_Phospholipids_VLDL.2 & 6.555 [5.123 7.707] & 2.465 [1.86 3.462] & 1.22 & $4.59 \times 10^{-5}$ & $3.27 \times 10^{-4}$ \\
\hline MainFractions_Phospholipids_IDL & 13.195 [11.277 13.99] & 4.77 [3.405 5.865] & 1.44 & $6.03 \times 10^{-5}$ & $4.04 \times 10^{-4}$ \\
\hline VLDL_Subfractions_FreeCholesterol_VLDL.1 & $6.905[6.438 .595]$ & $1.345[0.481 .855]$ & 2.52 & $7.68 \times 10^{-5}$ & $4.87 \times 10^{-4}$ \\
\hline MainFractions_FreeCholesterol_VLDL & 19.25 [17.398 23.538] & 7.74 [5.652 9.453] & 1.43 & $9.83 \times 10^{-5}$ & $5.90 \times 10^{-4}$ \\
\hline MainFractions_FreeCholesterol_IDL & $5.695[4.5058 .465]$ & $2.78[1.7773 .518]$ & 1.32 & $1.40 \times 10^{-4}$ & $6.73 \times 10^{-4}$ \\
\hline VLDL_Subfractions_FreeCholesterol_VLDL.2 & 3.625 [2.663 3.978] & $1.16[0.8451 .642]$ & 1.55 & $1.41 \times 10^{-4}$ & $6.73 \times 10^{-4}$ \\
\hline Main_Parameters_Cholesterol & 242.43 [216.257 251.153] & 186.395 [170.765 211.16] & 0.33 & $1.42 \times 10^{-4}$ & $6.73 \times 10^{-4}$ \\
\hline VLDL_Subfractions_Cholesterol_VLDL.3 & $7.59[6.3059 .242]$ & $2.66[1.964 .388]$ & 1.34 & $1.42 \times 10^{-4}$ & $6.73 \times 10^{-4}$ \\
\hline VLDL_Subfractions_FreeCholesterol_VLDL.4 & $3.19[2.5225 .088]$ & $1.405[1.0871 .878]$ & 1.38 & $1.42 \times 10^{-4}$ & $6.73 \times 10^{-4}$ \\
\hline VLDL_Subfractions_Triglycerides_VLDL.4 & $14.33[12.16$ 18.005] & $7.765[5.549 .773]$ & 0.96 & $1.98 \times 10^{-4}$ & $9.02 \times 10^{-4}$ \\
\hline VLDL_Subfractions_Phospholipids_VLDL.4 & $6.795[5.6488 .938]$ & $3.99[2.8654 .717]$ & 0.88 & $2.74 \times 10^{-4}$ & $1.20 \times 10^{-3}$ \\
\hline IDL_Particle_Number & 123.81 [97.71 174.377] & 72.05 [59.055 92.15] & 0.93 & $3.73 \times 10^{-4}$ & $1.32 \times 10^{-3}$ \\
\hline LDL.6_Particle_Number & 627.52 [479.947 741.995] & $346.56[249.407458 .607]$ & 0.82 & $3.73 \times 10^{-4}$ & $1.32 \times 10^{-3}$ \\
\hline MainFractions_Apo.B_IDL & $6.805[5.3759 .592]$ & $3.965[3.255 .067]$ & 0.93 & $3.73 \times 10^{-4}$ & $1.32 \times 10^{-3}$ \\
\hline LDL_Subfractions_Phospholipids_LDL.6 & 20.9 [18.147 23.673] & 14.04 [11.262 17.135] & 0.59 & $3.73 \times 10^{-4}$ & $1.32 \times 10^{-3}$ \\
\hline LDL_Subfractions_Apo.B_LDL.6 & 34.515 [26.395 40.81] & 19.06 [13.715 25.225] & 0.82 & $3.73 \times 10^{-4}$ & $1.32 \times 10^{-3}$ \\
\hline HDL_Subfractions_Triglycerides_HDL.4 & $4.755[3.9454 .992]$ & $2.58[2.4332 .852]$ & 0.77 & $3.73 \times 10^{-4}$ & $1.32 \times 10^{-3}$ \\
\hline VLDL_Subfractions_Cholesterol_VLDL.2 & 7.385 [5.69 7.815] & $2.935[2.0023 .798]$ & 1.24 & $3.95 \times 10^{-4}$ & $1.32 \times 10^{-3}$ \\
\hline VLDL_Subfractions_Phospholipids_VLDL.3 & 7.77 [6.45 8.297] & 3.11 [2.045 3.857] & 1.21 & $3.95 \times 10^{-4}$ & $1.32 \times 10^{-3}$ \\
\hline VLDL_Subfractions_Triglycerides_VLDL.3 & 21.9 [18.928 23.95] & 9.045 [5.5 11.13] & 1.15 & $4.41 \times 10^{-4}$ & $1.44 \times 10^{-3}$ \\
\hline LDL_Subfractions_Cholesterol_LDL.6 & 39.615 [33.542 44.188] & $24.98[18.98531 .68]$ & 0.68 & $5.04 \times 10^{-4}$ & $1.60 \times 10^{-3}$ \\
\hline VLDL_Subfractions_Phospholipids_VLDL.5 & $2.29[2.1382 .743]$ & 1.16 [0.915 1.9] & 0.87 & $6.12 \times 10^{-4}$ & $1.89 \times 10^{-3}$ \\
\hline VLDL_Subfractions_FreeCholesterol_VLDL.5 & $1.66\left[\begin{array}{lll}1.202 & 2.345]\end{array}\right]$ & $0.575[0.3020 .908]$ & 1.49 & $6.70 \times 10^{-4}$ & $2.01 \times 10^{-3}$ \\
\hline VLDL_Subfractions_Cholesterol_VLDL.4 & $7.425[5.768$ 11.15] & 4.54 [3.668 5.143] & 0.9 & $8.84 \times 10^{-4}$ & $2.58 \times 10^{-3}$ \\
\hline VLDL_Subfractions_Triglycerides_VLDL.5 & $3.69[3.2983 .907]$ & $2.235\left[\begin{array}{lll}1.852 & 2.918\end{array}\right]$ & 0.6 & $1.15 \times 10^{-3}$ & $3.28 \times 10^{-3}$ \\
\hline HDL_Subfractions_Triglycerides_HDL.3 & $2.645[2.5182 .85]$ & 1.61 [1.395 1.738] & 0.71 & $3.84 \times 10^{-3}$ & $1.07 \times 10^{-2}$ \\
\hline Apo.B100_Apo.A1 & $0.755[0.7050 .815]$ & $0.59[0.4420 .62]$ & 0.4 & $4.46 \times 10^{-3}$ & $1.21 \times 10^{-2}$ \\
\hline MainFractions_Triglycerides_HDL & 12.12 [10.488 13.635] & 7.795 [6.595 9.035] & 0.63 & $5.91 \times 10^{-3}$ & $1.57 \times 10^{-2}$ \\
\hline HDL_Subfractions_Apo.A2_HDL.3 & $6.88[6.227 .827]$ & $5.75[5.4626 .303]$ & 0.3 & $9.97 \times 10^{-3}$ & $2.58 \times 10^{-2}$ \\
\hline LDL_Subfractions_Triglycerides_LDL.6 & 6 [5.397 7.277] & $4.94[4.2155 .12]$ & 0.34 & $1.08 \times 10^{-2}$ & $2.74 \times 10^{-2}$ \\
\hline LDL_Subfractions_FreeCholesterol_LDL.6 & $8.035[6.6358 .848]$ & 5.59 [4.115 7.095] & 0.54 & $1.18 \times 10^{-2}$ & $2.92 \times 10^{-2}$ \\
\hline VLDL_Subfractions_Cholesterol_VLDL.5 & 1.88 [1.268 1.94] & 0.935 [0.682 1.532] & 0.73 & $1.28 \times 10^{-2}$ & $3.10 \times 10^{-2}$ \\
\hline MainFractions_Apo.A2_HDL & $34.53[34.01239 .838]$ & $31.145[28.98833 .998]$ & 0.21 & $1.51 \times 10^{-2}$ & $3.58 \times 10^{-2}$ \\
\hline HDL_Subfractions_FreeCholesterol_HDL.2 & $1.365[1.0051 .562]$ & $1.705[1.5782 .295]$ & -0.48 & $1.77 \times 10^{-2}$ & $4.12 \times 10^{-2}$ \\
\hline LDL.Chol_HDL.Chol & 2.05 [1.872 2.608] & 1.66 [1.365 1.948] & 0.35 & $2.20 \times 10^{-2}$ & $4.92 \times 10^{-2}$ \\
\hline HDL_Subfractions_Cholesterol_HDL.2 & $7.945[6.668 .887]$ & $10.025[8.42511 .535]$ & -0.32 & $2.20 \times 10^{-2}$ & $4.92 \times 10^{-2}$ \\
\hline Main_Parameters_Apo.A2 & 33.51 [31.752 39.38] & 30.085 [28.275 32.907] & 0.19 & $3.06 \times 10^{-2}$ & $6.46 \times 10^{-2}$ \\
\hline LDL_Subfractions_Triglycerides_LDL.4 & 1.215 [1.105 1.64] & 2.055 [1.572 2.675] & -0.75 & $3.06 \times 10^{-2}$ & $6.46 \times 10^{-2}$ \\
\hline LDL_Subfractions_FreeCholesterol_LDL.3 & $3.41[1.5253 .945]$ & $4.775[4.3835 .195]$ & -0.61 & $3.06 \times 10^{-2}$ & $6.46 \times 10^{-2}$ \\
\hline Main_Parameters_HDL.Chol & $52.805[51.4856 .24]$ & $60.32[55.98366 .567]$ & -0.19 & $3.58 \times 10^{-2}$ & $7.16 \times 10^{-2}$ \\
\hline MainFractions_Cholesterol_HDL & $52.805[51.4856 .24]$ & $60.32[55.98366 .567]$ & -0.19 & $3.58 \times 10^{-2}$ & $7.16 \times 10^{-2}$ \\
\hline HDL_Subfractions_Apo.A1_HDL.3 & $25.605[24.71730 .588]$ & 23 [22.125 27.023] & 0.17 & $3.58 \times 10^{-2}$ & $7.16 \times 10^{-2}$ \\
\hline LDL.5_Particle_Number & 300.27 [247.382 321.38] & 166.685 [140.99 271.045] & 0.53 & $4.17 \times 10^{-2}$ & $7.93 \times 10^{-2}$ \\
\hline LDL_Subfractions_Apo.B_LDL.5 & $16.515[13.60817 .677]$ & $9.165[7.75514 .91]$ & 0.53 & $4.17 \times 10^{-2}$ & $7.93 \times 10^{-2}$ \\
\hline HDL_Subfractions_Apo.A2_HDL.4 & 21.8 [19.265 23.615] & 16.905 [14.2 20.055] & 0.27 & $4.17 \times 10^{-2}$ & $7.93 \times 10^{-2}$ \\
\hline MainFractions_FreeCholesterol_HDL & 9.35 [9.21 10.613] & $11.74[9.86713 .873]$ & -0.26 & $4.33 \times 10^{-2}$ & $8.10 \times 10^{-2}$ \\
\hline LDL_Particle_Number & 1413.735 [1368.12 1703.253] & 1290.365 [1040.328 1393.905] & 0.27 & $4.84 \times 10^{-2}$ & $8.76 \times 10^{-2}$ \\
\hline MainFractions_Apo.B_LDL & 77.755 [75.245 93.675] & 70.965 [57.218 76.665] & 0.27 & $4.84 \times 10^{-2}$ & $8.76 \times 10^{-2}$ \\
\hline LDL_Subfractions_Cholesterol_LDL.5 & 22.305 [19.075 26.078] & 11.495 [8.82 21.198] & 0.57 & $6.43 \times 10^{-2}$ & $1.11 \times 10^{-1}$ \\
\hline LDL_Subfractions_FreeCholesterol_LDL.2 & $4.665[3.7455 .432]$ & $6.255[4.7156 .837]$ & -0.34 & $6.43 \times 10^{-2}$ & $1.11 \times 10^{-1}$ \\
\hline HDL_Subfractions_FreeCholesterol_HDL.1 & $3.275[2.4274 .258]$ & 4.425 [3.698 5.373] & -0.43 & $6.43 \times 10^{-2}$ & $1.11 \times 10^{-1}$ \\
\hline
\end{tabular}


LDL_Subfractions_Triglycerides_LDL.1 LDL_Subfractions_Phospholipids_LDL.2 HDL_Subfractions_Apo.A1_HDL.1 HDL_Subfractions_Apo.A2_HDL.2 LDL_Subfractions_Phospholipids_LDL.5 MainFractions_Phospholipids_HDL MainFractions_Triglycerides_LDL LDL_Subfractions_Triglycerides_LDL.2 HDL_Subfractions_Triglycerides_HDL.2 LDL_Subfractions_Cholesterol_LDL.2 HDL_Subfractions_Phospholipid_HDL.1 HDL_Subfractions_Phospholipid_HDL.2 LDL_Subfractions_Triglycerides_LDL.5 LDL_Subfractions_FreeCholesterol_LDL.5 LDL_Subfractions_Phospholipids_LDL.3 HDL_Subfractions_Cholesterol_HDL.1 HDL_Subfractions_Apo.A1_HDL.4 Main_Parameters_LDL.Chol LDL.2 Particle Number MainFractions_Cholesterol_LDL LDL_Subfractions_Apo.B_LDL.2 Main_Parameters_Apo.A1 HDL_Subfractions_Triglycerides_HDL.1 HDL_Subfractions_FreeCholesterol_HDL.3 LDL.3_Particle_Number

LDL_Subfractions_Apo.B_LDL.3

LDL_Subfractions_Cholesterol_LDL.3 HDL_Subfractions_Cholesterol_HDL.3 LDL_Subfractions_Triglycerides_LDL.3 MainFractions_Phospholipids_LDL HDL_Subfractions_Phospholipid_HDL.3 LDL_Subfractions_FreeCholesterol_LDL.4 LDL_Subfractions_Cholesterol_LDL.1 HDL_Subfractions_Apo.A1_HDL.2 HDL_Subfractions_Phospholipid_HDL.4 LDL.4_Particle_Number LDL_Subfractions_Phospholipids_LDL.4 LDL_Subfractions_Apo.B_LDL.4 LDL.1_Particle_Number LDL_Subfractions_Apo.B_LDL.1 MainFractions_FreeCholesterol_LDL HDL_Subfractions_Apo.A2_HDL.1 MainFractions_Apo.A1_HDL HDL_Subfractions_FreeCholesterol_HDL.4 LDL_Subfractions_Cholesterol_LDL.4 LDL_Subfractions_Phospholipids_LDL.1 LDL_Subfractions_FreeCholesterol_LDL.1 HDL_Subfractions_Cholesterol_HDL.4
6.215 [4.98 7.638]

$8.03[5.6989 .823]$

21.315 [17.885 26.622]

4.045 [3.553 4.988]

11.475 [10.037 13.707]

71.915 [67.455 74.508]

21.375 [19.322 24.075]

$1.91[1.802$ 2.067]

2.015 [1.69 2.253]

$15.645[10.51718 .652]$

18.775 [15.248 20.535]

13.235 [10.63 14.83]

3.56 [2.708 3.827]

5.4 [4.493 6.308]

$6.72[1.8258 .13]$

16.89 [13.783 19.642]

73.3 [63.96 83.575]

109.14 [99.135 134.655]

152.755 [114.168 179.565]

109.14 [99.135 134.655] 8.4 [6.28 9.872]

143.235 [136.41 150.05]

3.54 [2.73 3.958]

$1.68[1.3881 .87]$

131.705 [37.047 171.588]

7.245 [2.04 9.44]

11.685 [2.105 15.377]

8.86 [8.398 10.175]

2.395 [1.852 2.637]

59.635 [54.36 74.132]

15.095 [14.135 16.82]

3.065 [1.095 4.562]

18.885 [15.72 25.035]

17.805 [15.957 21.42]

26.26 [20.55 29.66]

132.31 [49.392 180.19]

5.705 [1.485 8.697]

7.275 [2.72 9.91]

202.365 [170.42 230.515]

11.13 [9.37 12.68]

28.15 [25.31 36.675]

2.71 [2.225 3.487]

140.92 [136.89 147.097]

3.055 [1.69 3.705]

10.105 [2.053 16.44]

10.87 [9.21 13.52]

5.19 [4.58 7.225]

19.02 [14.853 22.042]
4.66 [3.415 6.215]

10.935 [8.47 11.943]

33.085 [21.492 38.812]

3.53 [2.595 4.11]

6.905 [5.468 11.435]

$78.99[71.60886 .375]$

19.655 [15.92 20.8]

2.6 [1.895 3.11]

1.62 [1.118 1.89]

20.02 [14.985 21.427]

24.395 [19.26 30.763]

15.465 [12.505 17.177]

$2.725[2.223 .428]$

3.31 [2.975 5.9]

7.88 [5.605 8.828]

20.415 [15.768 26.862]

66.545 [59.858 75.243]

103.665 [83.118 113.803]

187.735 [143.328 209.08]

103.665 [83.118 113.803]

10.325 [7.88 11.495]

138.3 [133.245 148.077]

2.96 [1.878 3.848]

1.76 [1.452 2.138]

146.74 [108.203 174.567]

8.07 [5.95 9.598]

13.41 [8.697 15.675]

9.345 [8.625 10.555]

$2.475[1.7352 .86]$

59.185 [49.73 65.748]

14.365 [13.732 17.263]

3.54 [2.478 4.383]

20.14 [13.995 26.125]

18.075 [15.985 20.025]

23.26 [21.895 27.84]

126.205 [59.178 156.785]

6.23 [3.59 7.855]

6.945 [3.255 8.625]

209.91 [145.275 253.295]

11.545 [7.992 13.93]

29.46 [25.735 33.145]

2.98 [2.49 3.685]

142.275 [135.855 148.532] $2.53[2.23 .5]$

10.1 [5.402 13.578]

$11.94[8.56514 .76]$

6.675 [4.457 7.775]

18.695 [16.23 22.68]
0.41

$-0.36$

$8.41 \times 10^{-2}$

$8.41 \times 10^{-2}$

$8.41 \times 10^{-2}$

$9.50 \times 10^{-2}$

$9.56 \times 10^{-2}$

$1.22 \times 10^{-1}$

$1.38 \times 10^{-1}$

$1.43 \times 10^{-1}$

$1.43 \times 10^{-1}$

$1.54 \times 10^{-1}$

$1.54 \times 10^{-1}$

$1.54 \times 10^{-1}$

$1.69 \times 10^{-1}$

$1.88 \times 10^{-1}$

$1.92 \times 10^{-1}$

$1.92 \times 10^{-1}$

$2.35 \times 10^{-1}$

$2.59 \times 10^{-1}$

$2.59 \times 10^{-1}$

$2.59 \times 10^{-1}$

$2.59 \times 10^{-1}$

$3.12 \times 10^{-1}$

$3.41 \times 10^{-1}$

$3.96 \times 10^{-1}$

$4.03 \times 10^{-1}$

$4.03 \times 10^{-1}$

$4.29 \times 10^{-1}$

$4.72 \times 10^{-1}$

$5.78 \times 10^{-1}$

$5.85 \times 10^{-1}$

$6.25 \times 10^{-1}$

$6.82 \times 10^{-1}$

$7.52 \times 10^{-1}$

$8.38 \times 10^{-1}$

$8.41 \times 10^{-1}$

$8.61 \times 10^{-1}$

$8.61 \times 10^{-1}$

$8.61 \times 10^{-1}$

$8.86 \times 10^{-1}$

$8.86 \times 10^{-1}$

$9.07 \times 10^{-1}$

$9.07 \times 10^{-1}$

$9.31 \times 10^{-1}$

$9.31 \times 10^{-1}$

$9.53 \times 10^{-1}$

$9.53 \times 10^{-1}$

$9.77 \times 10^{-1}$

$9.77 \times 10^{-1}$
$1.39 \times 10^{-1}$

$1.39 \times 10^{-1}$

$1.39 \times 10^{-1}$

$1.54 \times 10^{-1}$

$1.54 \times 10^{-1}$

$1.94 \times 10^{-1}$

$2.15 \times 10^{-1}$

$2.18 \times 10^{-1}$

$2.18 \times 10^{-1}$

$2.25 \times 10^{-1}$

$2.25 \times 10^{-1}$

$2.25 \times 10^{-1}$

$2.43 \times 10^{-1}$

$2.66 \times 10^{-1}$

$2.66 \times 10^{-1}$

$2.66 \times 10^{-1}$

$3.23 \times 10^{-1}$

$3.39 \times 10^{-1}$

$3.39 \times 10^{-1}$

$3.39 \times 10^{-1}$

$3.39 \times 10^{-1}$

$4.04 \times 10^{-1}$

$4.36 \times 10^{-1}$

$5.00 \times 10^{-1}$

$5.00 \times 10^{-1}$

$5.00 \times 10^{-1}$

$5.26 \times 10^{-1}$

$5.72 \times 10^{-1}$

$6.94 \times 10^{-1}$

$6.95 \times 10^{-1}$

$7.35 \times 10^{-1}$

$7.93 \times 10^{-1}$

$8.66 \times 10^{-1}$

$9.43 \times 10^{-1}$

$9.43 \times 10^{-1}$

$9.43 \times 10^{-1}$

$9.43 \times 10^{-1}$

$9.43 \times 10^{-1}$

$9.53 \times 10^{-1}$

$9.53 \times 10^{-1}$

$9.57 \times 10^{-1}$

$9.57 \times 10^{-1}$

$9.65 \times 10^{-1}$

$9.65 \times 10^{-1}$

$9.70 \times 10^{-1}$

$9.70 \times 10^{-1}$

$9.77 \times 10^{-1}$

$9.77 \times 10^{-1}$ 


\section{$445 \quad$ References}

446 1. Bray F, Ferlay J, Soerjomataram I, Siegel RL, Torre LA, Jemal A. Global cancer statistics 2018: GLOBOCAN

447 estimates of incidence and mortality worldwide for 36 cancers in 185 countries. CA: a cancer journal for clinicians. $448 \quad 2018 ; 68(6): 394-424$.

449 2. Crawford ED. Epidemiology of prostate cancer. Urology. 2003;62(6 Suppl 1):3-12.

$4503 . \quad$ Powell IJ. The precise role of ethnicity and family history on aggressive prostate cancer: a review analysis. 451 Arch Esp Urol. 2011;64(8):711-9.

452 4. Tindall EA, Monare LR, Petersen DC, Van Zyl S, Hardie RA, Segone AM, et al. Clinical presentation of 453 prostate cancer in black South Africans. 2014;74(8):880-91.

454 5. Jaratlerdsiri W, Chan EK, Gong T, Petersen DC, Kalsbeek AM, Venter PA, et al. Whole-Genome Sequencing 455 Reveals Elevated Tumor Mutational Burden and Initiating Driver Mutations in African Men with Treatment-Naïve, 456 High-Risk Prostate Cancer. 2018;78(24):6736-46.

457 6. Dechambenoit G. Access to health care in sub-Saharan Africa. Surg Neurol Int. 2016;7:108.

458 7. Choudhury A, Ramsay M, Hazelhurst S, Aron S, Bardien S, Botha G, et al. Whole-genome sequencing for 459 an enhanced understanding of genetic variation among South Africans. Nature communications. 2017;8(1):1-12.

460 8. Patterson N, Petersen DC, van der Ross RE, Sudoyo H, Glashoff RH, Marzuki S, et al. Genetic structure of a 461 unique admixed population: implications for medical research. Human Molecular Genetics. 2010;19(3):411-9.

462 9. de Wit E, Delport W, Rugamika CE, Meintjes A, Möller M, van Helden PD, et al. Genome-wide analysis of 463 the structure of the South African Coloured Population in the Western Cape. Human genetics. 2010;128(2):14546453.

465 10. Amundadottir LT, Sulem P, Gudmundsson J, Helgason A, Baker A, Agnarsson BA, et al. A common variant 466 associated with prostate cancer in European and African populations. Nat Genet. 2006;38(6):652-8.

467 11. Blattner M, Lee DJ, O'Reilly C, Park K, MacDonald TY, Khani F, et al. SPOP mutations in prostate cancer 468 across demographically diverse patient cohorts. Neoplasia. 2014;16(1):14-20. 
469 12. Rand KA, Rohland N, Tandon A, Stram A, Sheng X, Do R, et al. Whole-exome sequencing of over 4100 men 470 of African ancestry and prostate cancer risk. Hum Mol Genet. 2016;25(2):371-81.

471 13. Huang FW, Mosquera JM, Garofalo A, Oh C, Baco M, Amin-Mansour A, et al. Exome Sequencing of

472 African-American Prostate Cancer Reveals Loss-of-Function ERF Mutations. Cancer Discov. 2017;7(9):973-83.

473 14. Devaney JM, Wang S, Furbert-Harris P, Apprey V, Ittmann M, Wang BD, et al. Genome-wide differentially 474 methylated genes in prostate cancer tissues from African-American and Caucasian men. Epigenetics.

$475 \quad 2015 ; 10(4): 319-28$.

476 15. Dreussi E, Ecca F, Scarabel L, Gagno S, Toffoli G. Immunogenetics of prostate cancer: a still unexplored 477 field of study. Pharmacogenomics. 2018;19(3):263-83.

478 16. Winchester DA, Till C, Goodman PJ, Tangen CM, Santella RM, Johnson-Pais TL, et al. Variation in genes 479 involved in the immune response and prostate cancer risk in the placebo arm of the Prostate Cancer Prevention 480 Trial. The Prostate. 2015;75(13):1403-18.

481 17. Stark T, Livas L, Kyprianou N. Inflammation in prostate cancer progression and therapeutic targeting. 482 Transl Androl Urol. 2015;4(4):455-63.

483 18. Batai K, Murphy AB, Nonn L, Kittles RA. Vitamin D and immune response: implications for prostate cancer 484 in African Americans. Frontiers in immunology. 2016;7:53.

485 19. Eastham JA, May RA, Whatley T, Crow A, Venable DD, Sartor O. Clinical characteristics and biopsy 486 specimen features in African-American and white men without prostate cancer. J Natl Cancer Inst. $487 \quad 1998 ; 90(10): 756-60$

488 20. Wallace TA, Prueitt RL, Yi M, Howe TM, Gillespie JW, Yfantis HG, et al. Tumor immunobiological 489 differences in prostate cancer between African-American and European-American men. Cancer Res.

$490 \quad 2008 ; 68(3): 927-36$.

491 21. Chechlinska M, Kowalewska M, Nowak RJNRC. Systemic inflammation as a confounding factor in cancer 492 biomarker discovery and validation. 2010;10(1):2. 
493 22. Bertini I, Cacciatore S, Jensen BV, Schou JV, Johansen JS, Kruhoffer M, et al. Metabolomic NMR

494 fingerprinting to identify and predict survival of patients with metastatic colorectal cancer. Cancer Res.

$495 \quad 2012 ; 72(1): 356-64$.

496 23. Cacciatore S, Loda M. Innovation in metabolomics to improve personalized healthcare. Ann N Y Acad Sci.

$497 \quad 2015 ; 1346(1): 57-62$.

498 24. Ritchie SC, Würtz P, Nath AP, Abraham G, Havulinna AS, Fearnley LG, et al. The biomarker GlycA is

499 associated with chronic inflammation and predicts long-term risk of severe infection. 2015;1(4):293-301.

500 25. Otvos JD, Shalaurova I, Wolak-Dinsmore J, Connelly MA, Mackey RH, Stein JH, et al. GlycA: A Composite

501 Nuclear Magnetic Resonance Biomarker of Systemic Inflammation. Clin Chem. 2015;61(5):714-23.

502 26. Gruppen EG, Connelly MA, Otvos JD, Bakker SJ, Dullaart RP. A novel protein glycan biomarker and LCAT 503 activity in metabolic syndrome. Eur J Clin Invest. 2015;45(8):850-9.

504 27. Ritchie SC, Würtz P, Nath AP, Abraham G, Havulinna AS, Fearnley LG, et al. The biomarker GlycA is 505 associated with chronic inflammation and predicts long-term risk of severe infection. Cell Syst 2015;1(4):293-301.

506 28. Gruppen EG, Connelly MA, Dullaart RP. Higher circulating GlycA, a pro-inflammatory glycoprotein 507 biomarker, relates to lipoprotein-associated phospholipase A2 mass in nondiabetic subjects but not in diabetic or 508 metabolic syndrome subjects. J Clin Lipidol. 2016;10(3):512-8.

509 29. Ritchie SC, Würtz P, Nath AP, Abraham G, Havulinna AS, Kangas AJ, et al. Systems medicine links microbial 510 inflammatory response with glycoprotein-associated mortality risk. Cell Syst. 2015:018655.

$51130 . \quad$ Gabay C, Kushner I. Acute-phase proteins and other systemic responses to inflammation. N Engl J Med. $512 \quad 1999 ; 340(6): 448-54$

513 31. Jiménez B, Holmes E, Heude C, Tolson RF, Harvey N, Lodge SL, et al. Quantitative lipoprotein subclass and 514 low molecular weight metabolite analysis in human serum and plasma by $1 \mathrm{H}$ NMR spectroscopy in a 515 multilaboratory trial. Anal Chem. 2018;90(20):11962-71. 
516 32. Rousseeuw PJ. Silhouettes: a graphical aid to the interpretation and validation of cluster analysis. Journal

517 of computational and applied mathematics. 1987;20:53-65.

518 33. Eriksson L, Jaworska J, Worth AP, Cronin MT, McDowell RM, Gramatica P. Methods for reliability and

519 uncertainty assessment and for applicability evaluations of classification- and regression-based QSARs. Environ

$520 \quad$ Health Perspect. 2003;111(10):1361-75.

521 34. Sciarra A, Gentilucci A, Salciccia S, Pierella F, Del Bianco F, Gentile V, et al. Prognostic value of

522 inflammation in prostate cancer progression and response to therapeutic: a critical review. J Inflamm (Lond).

$523 \quad 2016 ; 13: 35$

524 35. Ammirante M, Luo JL, Grivennikov S, Nedospasov S, Karin M. B-cell-derived lymphotoxin promotes 525 castration-resistant prostate cancer. Nature. 2010;464(7286):302-5.

526 36. Fuertes-Martin R, Correig X, Vallve JC, Amigo N. Title: Human Serum/Plasma Glycoprotein Analysis by

527 (1)H-NMR, an Emerging Method of Inflammatory Assessment. J Clin Med. 2020;9(2).

528 37. Lorenzo C, Festa A, Hanley AJ, Rewers MJ, Escalante A, Haffner SM. Novel Protein Glycan-Derived

529 Markers of Systemic Inflammation and C-Reactive Protein in Relation to Glycemia, Insulin Resistance, and Insulin

530 Secretion. Diabetes Care. 2017;40(3):375-82.

531 38. Reynolds AP, Richards G, de la Iglesia B, Rayward-Smith VJ. Clustering rules: a comparison of partitioning 532 and hierarchical clustering algorithms. Journal of Mathematical Modelling and Algorithms. 2006;5(4):475-504.

533 39. Sharma V, Freeze HH. Mannose Efflux from the Cells A POTENTIAL SOURCE OF MANNOSE IN BLOOD.

534 Journal of Biological Chemistry. 2011;286(12):10193-200.

535 40. Steed AL, Christophi GP, Kaiko GE, Sun L, Goodwin VM, Jain U, et al. The microbial metabolite 536 desaminotyrosine protects from influenza through type I interferon. 2017;357(6350):498-502.

537 41. Dewar M, Kaestner L, Zikhali Q, Jehle K, Sinha S, Lazarus J. Investigating racial differences in clinical and 538 pathological features of prostate cancer in South African men. South African Journal of Surgery. 2018;56(2):54-8. 
539 42. Niu YC, Feng RN, Hou Y, Li K, Kang Z, Wang J, et al. Histidine and arginine are associated with

540 inflammation and oxidative stress in obese women. Br J Nutr. 2012;108(1):57-61.

541 43. McGarrah RW, Kelly JP, Craig DM, Haynes C, Jessee RC, Huffman KM, et al. A Novel Protein Glycan-

542 Derived Inflammation Biomarker Independently Predicts Cardiovascular Disease and Modifies the Association of

543 HDL Subclasses with Mortality. Clin Chem. 2017;63(1):288-96.

544 44. Kim KI, Oh SW, Ahn S, Heo NJ, Kim S, Chin HJ, et al. CRP level and HDL cholesterol concentration jointly 545 predict mortality in a Korean population. Am J Med. 2012;125(8):787-95 e4.

546 45. Zewinger S, Drechsler C, Kleber ME, Dressel A, Riffel J, Triem S, et al. Serum amyloid A: high-density 547 lipoproteins interaction and cardiovascular risk. Eur Heart J. 2015;36(43):3007-16.

548 46. Arthur R, Møller H, Garmo H, Holmberg L, Stattin P, Malmstrom H, et al. Association between baseline 549 serum glucose, triglycerides and total cholesterol, and prostate cancer risk categories. 2016;5(6):1307-18.

550 47. Kotani K, Sekine Y, Ishikawa S, Ikpot IZ, Suzuki K, Remaley AT. High-density lipoprotein and prostate 551 cancer: an overview. J Epidemiol. 2013;23(5):313-9.

552 48. Allott EH, Howard LE, Cooperberg MR, Kane CJ, Aronson WJ, Terris MK, et al. Serum lipid profile and risk 553 of prostate cancer recurrence: Results from the SEARCH database. Cancer Epidemiol Biomarkers Prev.

$554 \quad 2014 ; 23(11): 2349-56$

555 49. Vaughan RA, Garcia-Smith R, Dorsey J, Griffith JK, Bisoffi M, Trujillo KAJljoc. Tumor necrosis factor alpha 556 induces Warburg-like metabolism and is reversed by anti-inflammatory curcumin in breast epithelial cells. $557 \quad 2013 ; 133(10): 2504-10$.

558 50. Vaughan RA, Garcia-Smith R, Trujillo KA, Bisoffi MJTP. Tumor necrosis factor alpha increases aerobic 559 glycolysis and reduces oxidative metabolism in prostate epithelial cells. 2013;73(14):1538-46.

560 51. Holroyde CP, Gabuzda TG, Putnam RC, Paul P, Reichard GAJCR. Altered glucose metabolism in metastatic 561 carcinoma. 1975;35(12):3710-4.

562 52. Shoelson SE, Lee J, Goldfine ABJTJoci. Inflammation and insulin resistance. 2006;116(7):1793-801. 
563 53. Llop E, Ferrer-Batallé M, Barrabés S, Guerrero PE, Ramírez M, Saldova R, et al. Improvement of prostate 564 cancer diagnosis by detecting PSA glycosylation-specific changes. Theranostics. 2016;6(8):1190.

565 54. Ferrer-Batallé M, Llop E, Ramírez M, Aleixandre RN, Saez M, Comet J, et al. Comparative study of blood566 based biomarkers, $\alpha 2$, 3-sialic acid PSA and PHI, for high-risk prostate cancer detection. International journal of 567 molecular sciences. 2017;18(4):845.

568 55. Ishikawa T, Yoneyama T, Tobisawa Y, Hatakeyama S, Kurosawa T, Nakamura K, et al. An automated micro569 total immunoassay system for measuring cancer-associated $\alpha 2,3$-linked sialyl $\mathrm{N}$-glycan-carrying prostate-specific 570 antigen may improve the accuracy of prostate cancer diagnosis. International journal of molecular sciences. $571 \quad 2017 ; 18(2): 470$

572 56. Labbé DP, Zadra G, Yang M, Reyes JM, Lin CY, Cacciatore S, et al. High-fat diet fuels prostate cancer 573 progression by rewiring the metabolome and amplifying the MYC program. Nature communications.

$574 \quad 2019 ; 10(1): 1-14$

575 57. Kristal AR, Arnold KB, Neuhouser ML, Goodman P, Platz EA, Albanes D, et al. Diet, supplement use, and 576 prostate cancer risk: results from the prostate cancer prevention trial. American journal of epidemiology. $577 \quad 2010 ; 172(5): 566-77$. 


\section{Figures}
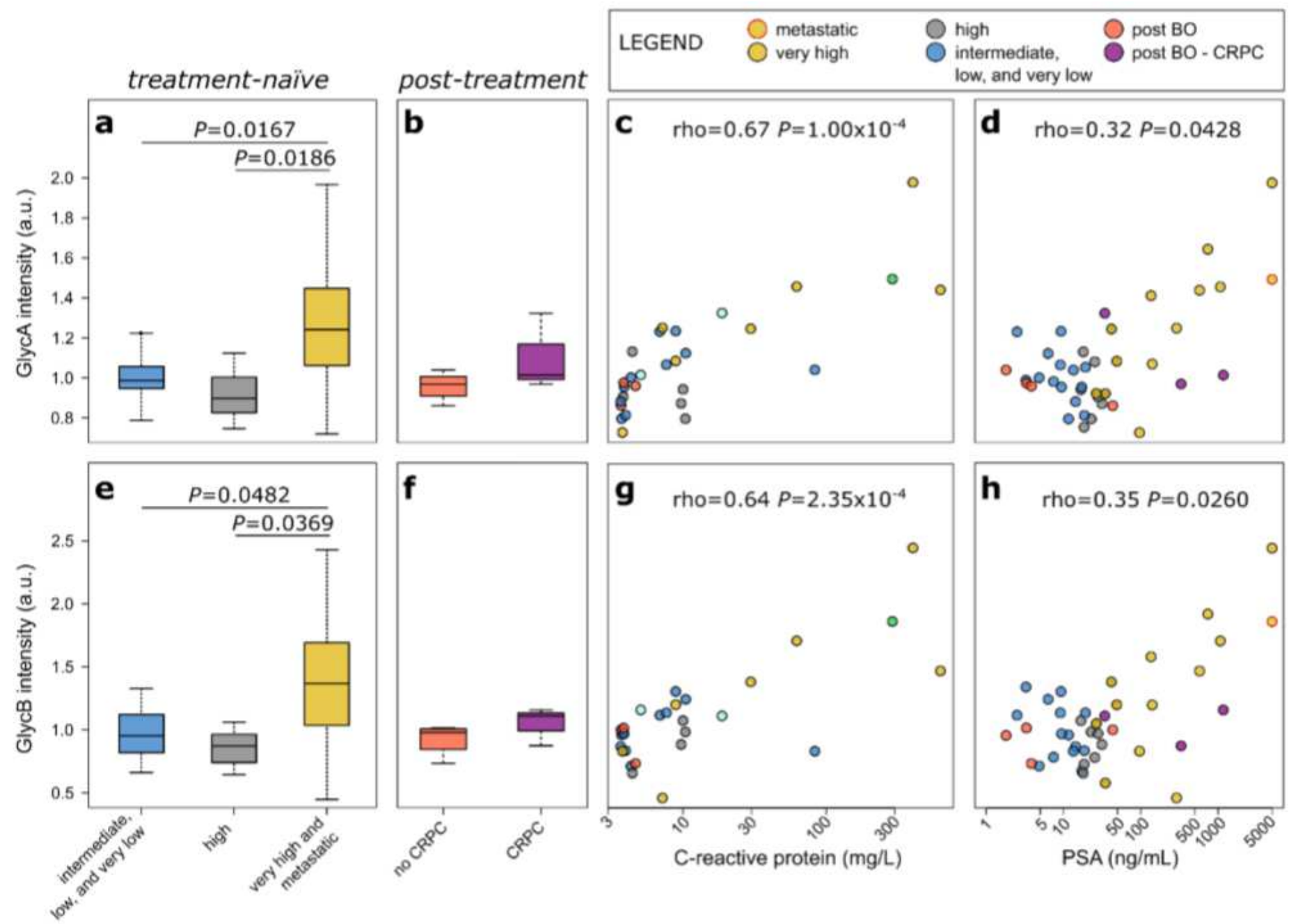

Figure 1

GlycA concentration in (a) treatment-naïve and (b) post-treatment. (c) Correlation between GlycA and CRP. (d) Correlation between GlycA and PSA. GlycB concentration in (e) treatment-naïve and (f) post-treatment. (g) Correlation between GlycB and CRP. (h) Correlation between GlycA and PSA. 

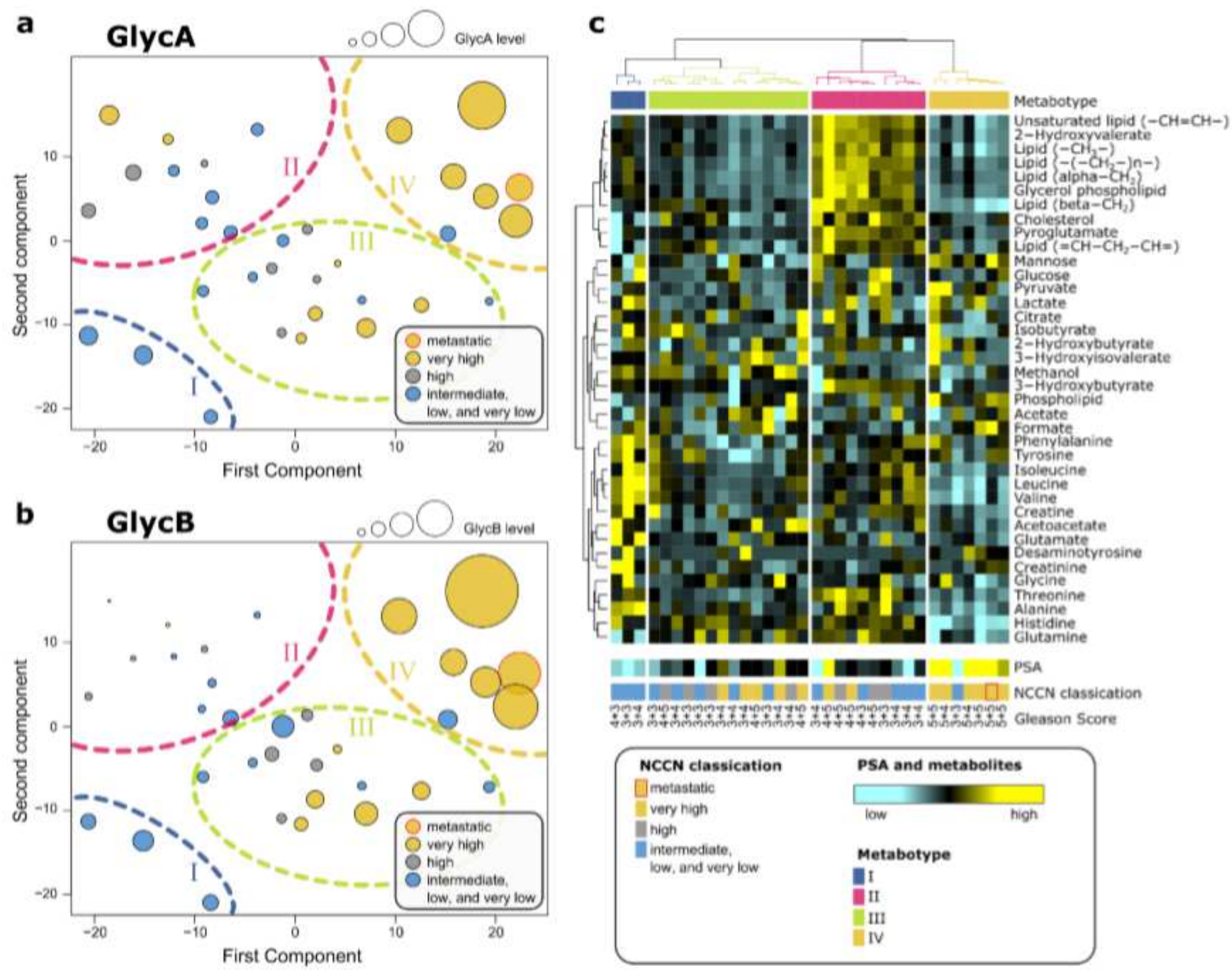

Figure 2

KODAMA score plot of plasma PCa samples colored according to NCCN classification. The size is proportional to (a) the GlycA intensity and (b) the GlycB intensity. (c) Heatmap of the metabolic profiles. 


\section{a}

NCCN classification

$\square$ metastatic
$\square$ very high
$\square$ high
$\square$ intermediate,
low, and very low

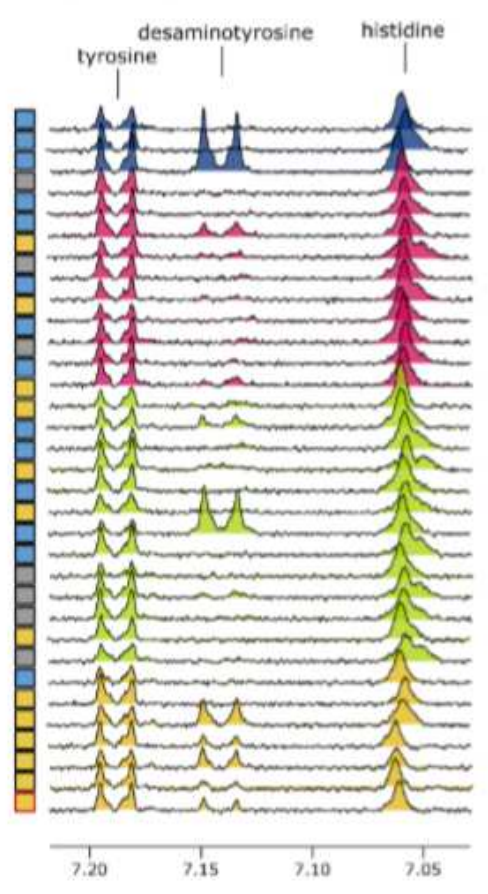

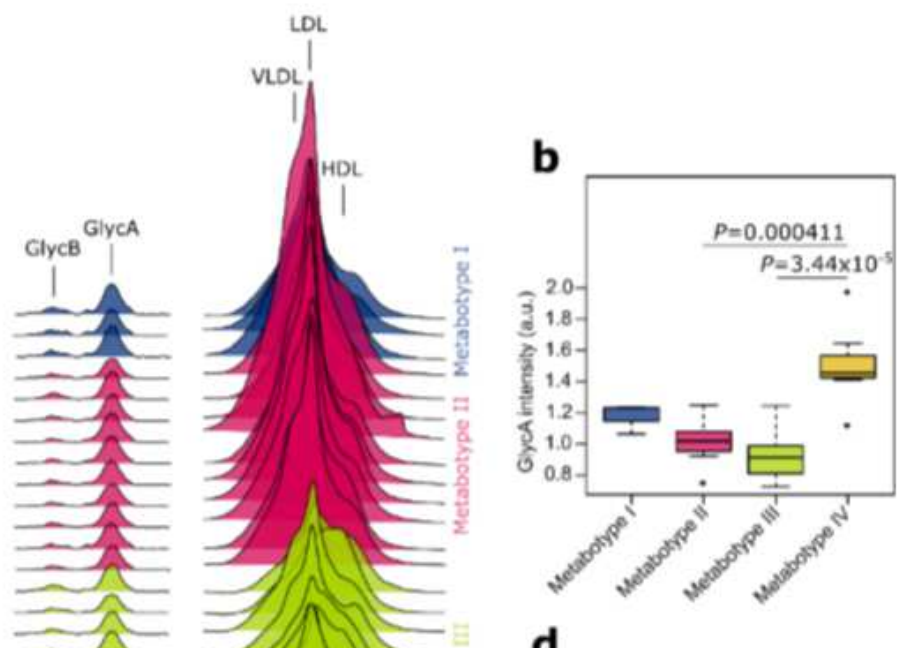

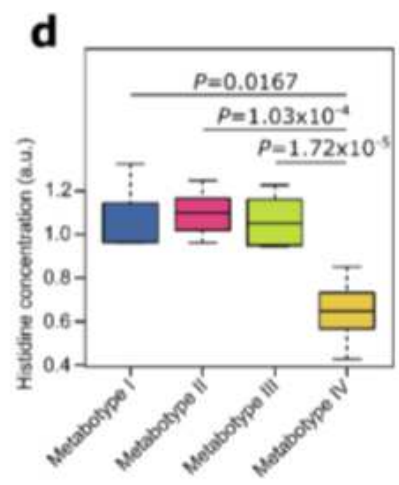

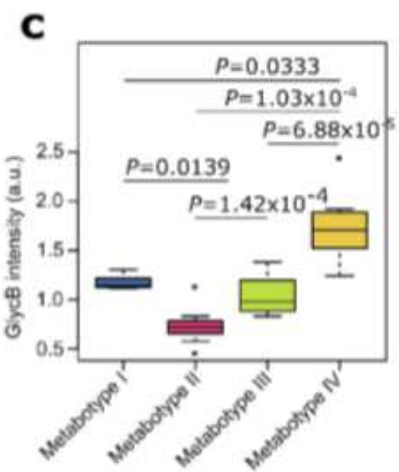

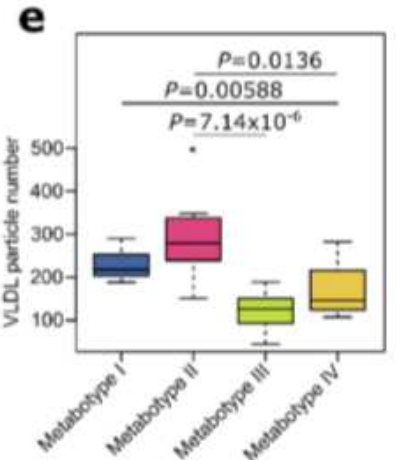

Chemical shift (ppm)

\section{Figure 3}

(a) NMR profiles of the plasma of three different spectral regions and Box-whiskers plots of the intensity of (b) GlycA and (c) GlycB, the concentration of (d) histidine and the number of (e) VLDL particles across the four metabotypes. 


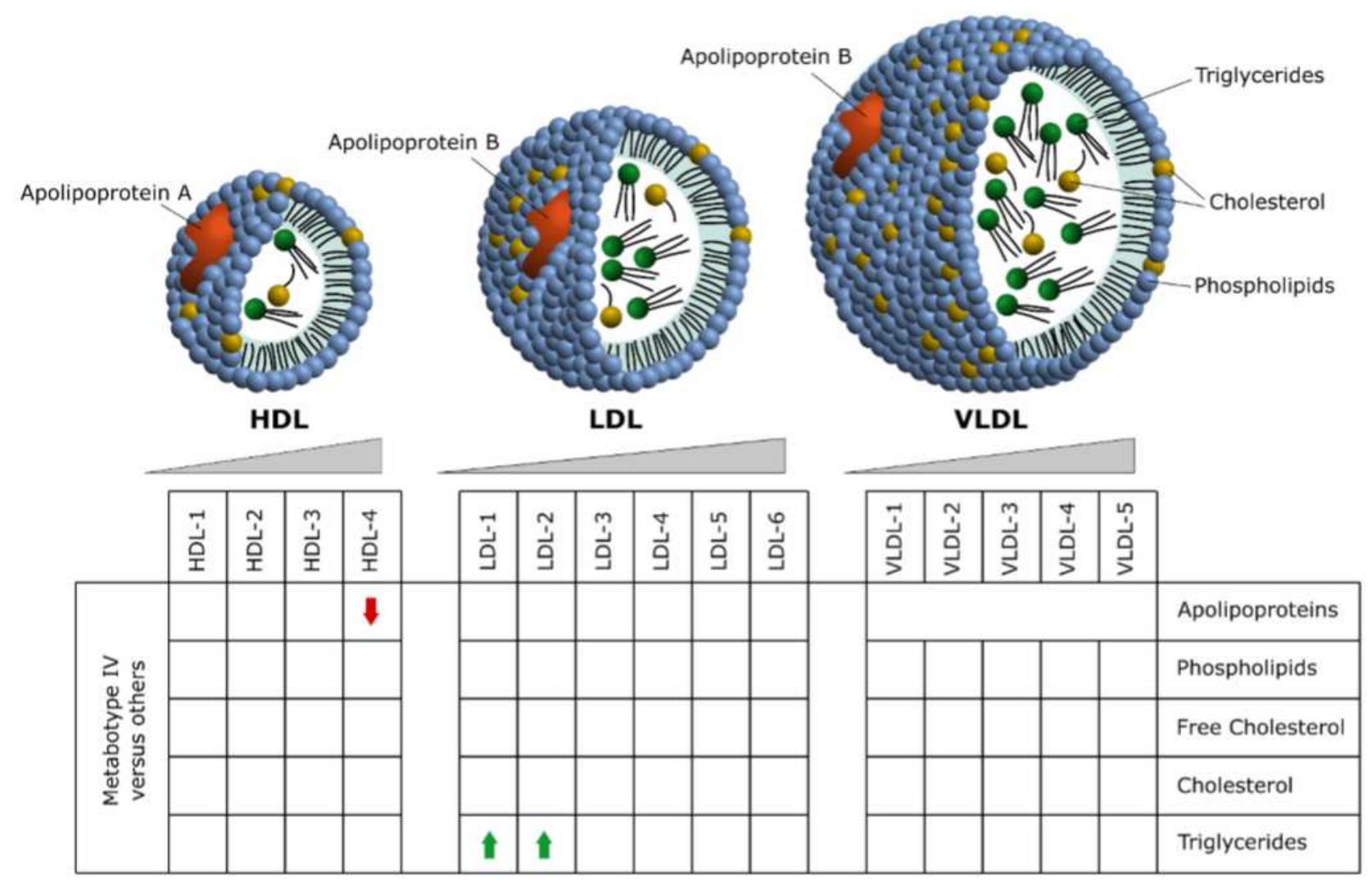

\begin{tabular}{|c|c|c|c|c|c|c|c|c|c|c|c|c|c|}
\hline \multirow{5}{*}{ 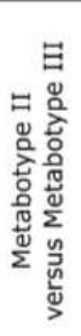 } & & t & | & & & 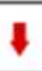 & t|t & \multicolumn{5}{|c|}{ ttet } & Apolipoproteins \\
\hline & & & & & & & tt & tot t| & tt|t & tt & t|t & t|t & Phospholipids \\
\hline & $\downarrow$ & & & $\downarrow$ & & & $\mathbf{1}$ & trt & t1t & tht & tt & t|t & Free Cholesterol \\
\hline & & & & & & & tet & tret & tÂt & têt & th & 1 & Cholesterol \\
\hline & & 1 & |1t & & $\downarrow$ & & 1 & tet & trt & t|t & tt & | & Triglycerides \\
\hline
\end{tabular}

Lower: $0.001<$ p-value $<0.01$

Higher: $0.001<p$-value $\leq 0.01$

1f $0.0001<\mathrm{p}$-value $\leq 0.001$

솟ㅅㄹ $0.00001<\mathrm{p}$-value $\leq 0.0001$

Figure 4

Graphics illustration of the changes of the lipoprotein profile. 